\title{
Yang-Baxterizations, Universal Quantum Gates and Hamiltonians
}

\author{
Yong Zhang ${ }^{1}$ \\ Institute of Theoretical Physics, Chinese Academy of Sciences \\ P. O. Box 2735, Beijing 100080, P. R. China \\ Louis H. Kauffman ${ }^{2}$ \\ Department of Mathematics, Statistics and Computer Science \\ University of Illinois at Chicago \\ 851 South Morgan Street, Chicago, IL, 60607-7045 \\ Mo-Lin $\mathrm{Ge}^{3}$ \\ Nankai Institute of Mathematics, Nankai University \\ Tianjin 300071, P. R. China
}

\begin{abstract}
The unitary braiding operators describing topological entanglements can be viewed as universal quantum gates for quantum computation. With the help of the Brylinski's theorem, the unitary solutions of the quantum Yang-Baxter equation can be also related to universal quantum gates. This paper derives unitary solutions of the quantum Yang-Baxter equation via Yang-Baxterization from the solutions of the braid relation. We study Yang-Baxterizations of the non-standard and standard representations of the six-vertex model and the complete solutions of the non-vanishing eight-vertex model. We construct Hamiltonians responsible for the time-evolution of the unitary braiding operators which lead to the Schrödinger equations.
\end{abstract}

Key Words: Topological entanglement, Quantum entanglement, Yang-Baxter, Universal quantum gates

PACS numbers: 02.10.Kn, 03.65.Ud, 03.67.Lx

\footnotetext{
${ }^{1}$ yong@itp.ac.cn

${ }^{2}$ kauffman@uic.edu

${ }^{3}$ GEML@nankai.edu.cn
} 


\section{Introduction}

There are natural relationships between quantum entanglement [1] and topological entanglement 2]. Topology studies global relationships in spaces, and how one space can be placed within another, such as knotting and linking of curves in three-dimensional space. One way to study topological entanglement and quantum entanglement is to try making direct correspondences between patterns of topological linking and entangled quantum states. One approach of this kind was initiated by Aravind [3], suggesting that observation of a link would be modelled by deleting one component of the link. But this correspondence property of quantum states and topological links is not basis independent 3 .

A deeper method (we believe) is to consider unitary gates $\check{R}$ that are both universal for quantum computation and are also solutions to the condition for topological braiding. Such $\check{R}$-matrices are unitary solutions to the Yang-Baxter equation (the braid relation). We are then in a position to compare the topological and quantum properties of these transformations. In this way, we can explore the apparently complex relationship among topological entanglement, quantum entanglement, and quantum computational universality. This way has been explored in a series of papers [4, 5, 6, 7, 8, 9, 10, 11, 12.

The present paper derives unitary solutions of the Quantum Yang-Baxter Equation (QYBE) via Yang-Baxterization and explores the corresponding dynamical evolution of quantum entanglement states. The solutions to the QYBE that we derive by Yang-Baxterization contain a spectral parameter $x$, and hence do not, except in special cases, give representations of the Artin braid group. These new solutions are unitary, and they do give useful quantum gates. Thus we show in this paper that the full physical subject of solutions to the quantum Yang-Baxter equation (including the spectral parameter $x$ ) is of interest for quantum computing and quantum information theory.

The plan of the paper is organized as follows. In the second section, a unitary braiding operator is regarded as a quantum entanglement operator and further a universal quantum gate. To describe the dynamical evolution of the unitary braiding operator $\check{R}$, Yang-Baxterization is used to solve the QYBE. In the third section, the unitary solutions of the QYBE for the non-standard and standard representations of the six-vertex model are obtained via Yang-Baxterization. In the fourth section, the complete unitary solutions of the QYBE for the nonvanishing eight-vertex model are obtained via Yang-Baxterization. In the fifth section, with the Brylinksi's theorem [13, all unitary $\check{R}(x)$-matrices presented in this paper are recognized to be universal quantum gates for most $x$-values. In the

sixth section, the Hamiltonian determining the time evolution of quantum state is constructed with the unitary $\check{R}(x)$-matrix. In the seventh section, as an example, the CNOT gate is constructed in terms of the unitary $\check{R}$-matrix $(\check{R}(x)$-matrix) and local unitary transformations. To conclude, remarks on our work are made. In Appendix A, a pragmatic introduction to Yang-Baxterization is presented. 


\section{The QYBE in quantum entanglements}

This section presents basic elements underlying our work. The Braid Group Representation (BGR) and the QYBE are described in the sense of studying quantum entanglements. The Brylinski's theorem [13 plays the key role in relating a unitary $\check{R}$-matrix $(\check{R}(x)$-matrix) to a universal quantum gate. Yang-Baxterization derives the corresponding $\check{R}(x)$-matrix from the $\check{R}$-matrix and makes it possible constructing the Hamiltonian determining the evolution of the unitary braiding operator ( $\check{R}$-matrix).

\subsection{The BGR in quantum entanglements}

Braids are patterns of entangled strings. A braid has the form of a collection of strings extending from one set of points to another, with a constant number of points in each cross section. Braids start in one row of points and end in another. As a result, one can multiply two braids to form a third braid by attaching the end points of the first braid to the initial points of the second braid. Up to topological equivalence, this multiplication gives rise to a group, the Artin braid group $B_{n}$ on $n$ strands, which is generated by $\left\{b_{i} \mid 1 \leq i \leq n-1\right\}$. tions:

The group $B_{n}$ consists of all words of the form $b_{j_{1}}^{ \pm 1} b_{j_{2}}^{ \pm 1} \ldots b_{j_{n}}^{ \pm 1}$ modulo the rela-

$$
\begin{aligned}
b_{i} b_{i+1} b_{i} & =b_{i+1} b_{i} b_{i+1}, \quad 1 \leq i \leq n-1, \\
b_{i} b_{j} & =b_{j} b_{i}, \quad|i-j|>1 .
\end{aligned}
$$

Each braid is, in itself, a pattern of entanglement. Each braid is also an operator that operates on other patterns of entanglement (braids) to produce new entanglements (braids again).

The analogy between topological entanglement and quantum entanglement (from the point of view of braids) means the association of a unitary operator with a braid that respects the topological structure of the braid and allows exploration of the entanglement properties of the operator. In other words, we propose to study the analogy between topological entanglement and quantum entanglement by looking at unitary representations of the Artin braid group. The main point for the exploration of the analogy is that (from the point of view of a BGR) each braid is seen as an operator rather than a state. See Fig. 1.

Consider representations of the Artin braid group constructed in the following manner. To an elementary two strand braid there is associated an operator

$$
\check{R}: V \otimes V \longrightarrow V \otimes V .
$$

Here $V$ is a complex vector space, and for our purposes, $V$ will be two dimensional so that $V$ can hold a single qubit of information. One should think of the two input and two output lines from the braid as representing this map of tensor products. Thus the left endpoints of $\check{R}$ as shown in Fig. 1 and Fig. 2 represent 
the tensor product $V \otimes V$ that forms the domain of $\check{R}$ and the right endpoints of the diagram for $\check{R}$ represent $V \otimes V$ as the range of the mapping. In the diagrams with three lines shown in Fig. 2, we have mappings from $V \otimes V \otimes V$ to itself.

The identity shown in Fig. 2 is called the Yang-Baxter Equation (the braid relation), and it reads algebraically as follows, where $I d$ denotes the identity transformation on $V$.

$$
(\check{R} \otimes I d)(I d \otimes \check{R})(\check{R} \otimes I d)=(I d \otimes \check{R})(\check{R} \otimes I d)(I d \otimes \check{R})
$$

This equation expresses the fundamental topological relation in the Artin braid group, and is the main requirement for producing a representation of the braid group by this method.

\subsection{The Brylinski's theorem and $\check{R}$-matrix}

A two-qubit gate $G$ is a unitary linear mapping $G: V \otimes V \longrightarrow V \otimes V$ where $V$ is a two complex dimensional vector space. A gate $G$ is said to be entangling if there is a vector

$$
|\alpha \beta\rangle=|\alpha\rangle \otimes|\beta\rangle \in V \otimes V
$$

such that $G|\alpha \beta\rangle$ is not decomposable as a tensor product of two qubits. Under these circumstances, one says that $G|\alpha \beta\rangle$ is entangled.

In [13, the Brylinskis give a general criterion of $G$ to be universal (in the presence of local unitary transformations). They prove that a two-qubit gate $G$ is universal if and only if it is entangling.

Here is the specific $\check{R}$-matrix that we shall examine

$$
\check{R}=\left(\begin{array}{cccc}
a & 0 & 0 & 0 \\
0 & 0 & d & 0 \\
0 & c & 0 & 0 \\
0 & 0 & 0 & b
\end{array}\right)
$$

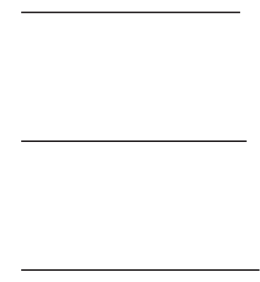

$I d \otimes I d \otimes I d$
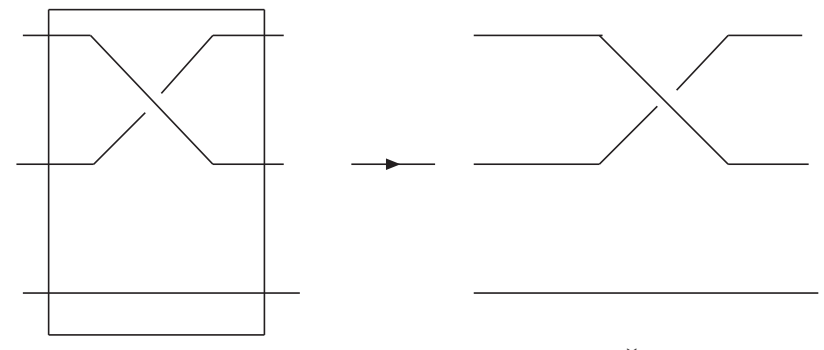

$\check{R} \otimes I d$

Figure 1: A braiding operator $\check{R} \otimes I d$. 

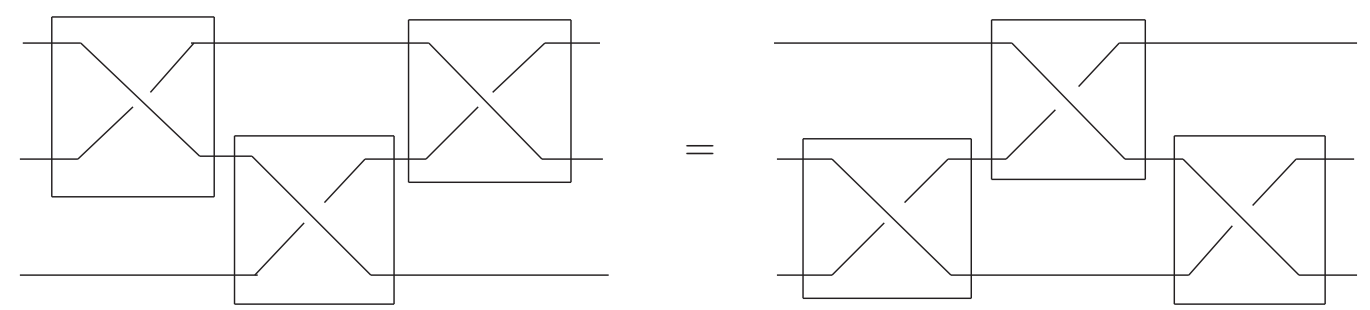

$(\check{R} \otimes I d)(I d \otimes \check{R})(\check{R} \otimes I d)=(I d \otimes \check{R})(\check{R} \otimes I d)(I d \otimes \check{R})$

Figure 2: The Yang-Baxter equation (the braid relation).

where $a, b, c, d$ can be any scalars on the unit circle in the complex plane. Then $\check{R}$ is a unitary matrix and it is a solution to the Yang-Baxter equation (3).

The point of this case study is that $\check{R}$, being unitary, can be considered as a universal quantum gate and since $\check{R}$ is the key ingredient in a unitary representation of the braid group, it can be considered as an operator that performs topological entanglement. We shall see that it can also perform quantum entanglement in its action on quantum states. The $\check{R}$-matrix can also be used to make an invariant of knots and links that is sensitive to linking numbers [2].

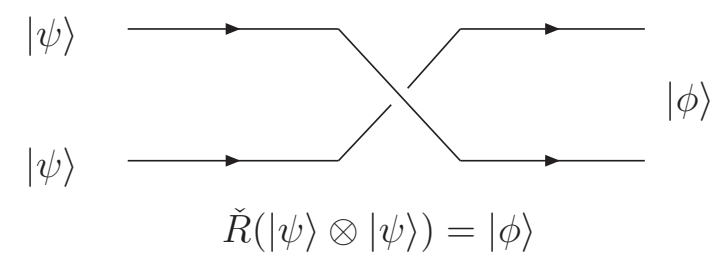

Figure 3: The braiding operator $\check{R}$ as an entangling operator.

Consider the action of the unitary transformation $\check{R}$ on quantum states. We have

$$
\begin{array}{rlrl}
\check{R}|00\rangle & =a|00\rangle, & & \check{R}|01\rangle=c|10\rangle, \\
\check{R}|10\rangle=d|01\rangle, & & \check{R}|11\rangle=b|11\rangle .
\end{array}
$$

Here is an elementary proof that the operator $\check{R}$ can entangle quantum states. If $\check{R}$ is chosen so that $a b \neq c d$, then the state $\check{R}(|\psi\rangle \otimes|\psi\rangle)$, with $|\psi\rangle=|0\rangle+|1\rangle$, is entangled as a quantum state since

$$
|\phi\rangle=\check{R}(|\psi\rangle \otimes|\psi\rangle)=a|00\rangle+c|10\rangle+d|01\rangle+b|11\rangle
$$

for $a b \neq c d$ (see Fig. 3) is an entangled state. 


\subsection{Yang-Baxterization and Hamiltonian}

In this paper, the BGR b-matrix [5] and the QYBE solution $\check{R}$-matrix [14, 15. 16, 17. are $n^{2} \times n^{2}$ matrices acting on $V \otimes V$ where $V$ is an $n$-dimensional vector space. As $b$ and $\check{R}$ act on the tensor product $V_{i} \otimes V_{i+1}$, we denote them by $b_{i}$ and $\check{R}_{i}$, respectively.

The BGR $b$-matrix has to satisfy the braid relation

$$
b_{i} b_{i+1} b_{i}=b_{i+1} b_{i} b_{i+1},
$$

while the QYBE has the form

$$
\check{R}_{i}(x) \check{R}_{i+1}(x y) \check{R}_{i}(y)=\check{R}_{i+1}(y) \check{R}_{i}(x y) \check{R}_{i+1}(x)
$$

with the asymptotic condition

$$
\check{R}(x=0)=b,
$$

and $x$ called the spectral parameter. From these two equations both $b$ and $\check{R}(x)$ are fixed up to an overall scalar factor.

In terms of the permutation operator $P$ specified by $P(\xi \otimes \eta)=\eta \otimes \xi$ and the $\check{R}(x)$-matrix, the solution of the algebraic QYBE reading

$$
R_{12}(x) R_{13}(x y) R_{23}(y)=R_{23}(y) R_{13}(x y) R_{12}(x)
$$

takes the form

$$
R(x)=P \check{R}(x)
$$

where $R_{i j}$ is an operator acting on the tensor product $V_{i} \otimes V_{j}$. But we will mainly deal with the unitary $4 \times 4 \check{R}(x)$-matrix since the unitary permutation operator $P$ can be found in order to obtain the unitary $R(x)$-matrix.

As a solution of the QYBE (8), the $\check{R}(x)$-matrix usually depends on a deformation parameter $q$ and the spectral parameter $x$. With two such parameters, there exist two approaches to solving the QYBE (8). Taking the limit as $q \rightarrow 1$ leads to the classical $r$-matrix satisfying the classical Yang-Baxter equation. Then $q$ deforming $r$-matrices as solutions of the classical Yang-Baxter equation regain the q-dependence for the $\check{R}$-matrices as solutions for the QYBE (8). The QYBE solution via this line of thought has been systematized by a general strategy in quantum groups (Hopf algebras) approach [18, 19, 20, 21, 22, 23.

Taking the limit as $x \rightarrow 0$ leads to the braid relation (7) from the QYBE (8) and the BGR $b$-matrix from the $\check{R}(x)$-matrix. Concerning relations between the BGR and $x$-dependent solutions of the QYBE (8), we either reduce a known $\check{R}(x)$-matrix to the BGR $b$-matrix, see [25, 26, 27], or construct the $\check{R}(x)$-matrix from a given BGR $b$-matrix. Such a construction is called Yang-Baxterization.

In knot theory, these solutions were first studied by Jones [24] and Turaev [27] for the BGR (77) satisfying the Hecke algebra relations and for the BGR (7) satisfying the Birman-Wenzl algebra relations (corresponding to the Kauffman 
two-variable polynomial, see [2]). Later the more general cases with a BGR (7) having three or four unequal eigenvalues were considered [28, 29, 30, including all known trigonometric solutions to the QYBE (8). Also Yang-Baxterization of non-standard BGR $b$-matrix has been discussed 31, 32, 33, 34, 35, 36, 37, 38.

In this paper, via Yang-Baxterization the unitary $\check{R}(x)$-matrices are derived and used to construct Hamiltonians determining the time evolution of quantum states. In Appendix A, a pragmatic revisit to Yang-Baxterization has been given.

\section{Unitary $\check{R}(x)$-matrix: the six-vertex model}

In this section, we deal with Yang-Baxterizations of both the standard representation and the non-standard representation of the six-vertex model in detail, as an example of deriving the unitary $\check{R}(x)$-matrix with the spectral parameter $x$ from the given BGR $b$-matrix.

Consider a non-standard BGR $b$-matrix suitable for constructing the Alexander polynomial [2]

$$
b=\left(\begin{array}{cccc}
q & 0 & 0 & 0 \\
0 & 0 & 1 & 0 \\
0 & 1 & q-q^{-1} & 0 \\
0 & 0 & 0 & -q^{-1}
\end{array}\right)
$$

where the deformation parameter $q$ has been assumed to be non-vanishing. Let $b^{\dagger}$ the transpose and conjugation of $b$. The unitarity condition $b^{\dagger} b=b^{\dagger} b=\mathbb{1}$ leads to $q= \pm 1$.

It has two distinct eigenvalues: $q$ and $-q^{-1}$. By Yang-Baxterization as in Appendix A.1, the BGR $b$-matrix corresponds to the $\check{R}(x)$-matrix

$$
\check{R}(x)=\left(\begin{array}{cccc}
q-q^{-1} x & 0 & 0 & 0 \\
0 & \left(q-q^{-1}\right) x & 1-x & 0 \\
0 & 1-x & q-q^{-1} & 0 \\
0 & 0 & 0 & q x-q^{-1}
\end{array}\right)
$$

which satisfies the QYBE (8). The case of $x=1$ will not be considered since $\check{R}(1)=\left(q-q^{-1}\right) \mathbb{1}$.

Assume that $q, x$ are complex numbers, $x \neq 1$ and $q \neq 0$, with the complex conjugations $\bar{q}, \bar{x}$ and the norms $\|q\|^{2}:=q \bar{q},\|x\|^{2}:=x \bar{x}$. The $\check{R}(x)$-matrix has its conjugate matrix by

$$
\check{R}^{\dagger}(\bar{x})=\left(\begin{array}{cccc}
\bar{q}-\bar{q}^{-1} \bar{x} & 0 & 0 & 0 \\
0 & \left(\bar{q}-\bar{q}^{-1}\right) \bar{x} & 1-\bar{x} & 0 \\
0 & 1-\bar{x} & \bar{q}-\bar{q}^{-1} & 0 \\
0 & 0 & 0 & \bar{q} \bar{x}-\bar{q}^{-1}
\end{array}\right) .
$$

The unitarity condition shows

$$
\check{R}(x) \check{R}^{\dagger}(\bar{x})=\check{R}^{\dagger}(\bar{x}) \check{R}(x)=\rho \mathbb{1}
$$


where $\rho$ is a normalization factor of the $\check{R}(x)$-matrix. It gives us the following equations

$$
\left\{\begin{array}{ccc}
\left\|q-q^{-1} x\right\|^{2}=\left\|q x-q^{-1}\right\|^{2} & = & \rho \\
(1-x)\left(\bar{q}-\bar{q}^{-1}\right) \bar{x}+\left(q-q^{-1}\right)(1-\bar{x}) & = & 0 \\
\left\|q-q^{-1}\right\|^{2}\|x\|^{2}+\|1-x\|^{2} & = & \rho \\
\left\|q-q^{-1}\right\|^{2}+\|1-x\|^{2} & = & \rho
\end{array} .\right.
$$

Comparing the last two equations, we obtain

$$
\frac{1}{\|q\|^{2}}\left\|1-q^{2}\right\|^{2}\left(1-\|x\|^{2}\right)=0
$$

which leads to the first case of $q= \pm 1$ and the second case of $\|x\|=1$. With $\|x\|=1$ and

$$
q^{-1}=\frac{\bar{q}}{\|q\|^{2}}, \quad \bar{q}^{-1}=\frac{q}{\|q\|^{2}}
$$

going further to rewrite the above second equation as

$$
(1-\bar{x})\left(1+\frac{1}{\|q\|^{2}}\right)(q-\bar{q})=0
$$

which shows that $q$ has to be real. Analyzing the above first equation gives

$$
\frac{1}{\|q\|^{2}}(q+\bar{q})(q-\bar{q})(x-\bar{x})=0
$$

which is satisfied for real $q$. To make a safe judgement, comparing the first equation and the last two equations, we have

$$
(q-\bar{q})(q(1-\bar{x})+\bar{q}(1-x))=0,
$$

which is also satisfied for real $q$. Therefore, as $\|x\|=1, x \neq 1$ and $q$ real, the $\check{R}(x)$-matrix obtained through Yang-Baxterization is unitary.

In the first case that $q= \pm 1, x \neq 1$, we have

$$
\rho=\|1-x\|^{2}:=(1-x)(1-\bar{x}),
$$

and then the corresponding $\check{R}(x)$-matrix is given by

$$
\left.\check{R}(x)\right|_{q= \pm 1}=\left.\frac{1-x}{\|1-x\|} b\right|_{q= \pm 1} .
$$

In the second case, taking $x=e^{2 i \theta}, \theta \neq 0$ and $q=e^{\gamma}$, the $\check{R}(x)$-matrix takes the form

$$
\check{R}(\theta)=2 e^{i \theta}\left(\begin{array}{cccc}
\sinh (\gamma-i \theta) & 0 & 0 & 0 \\
0 & e^{i \theta} \sinh \gamma & -i \sin \theta & 0 \\
0 & -i \sin \theta & e^{-i \theta} \sinh \gamma & 0 \\
0 & 0 & 0 & \sinh (\gamma+i \theta)
\end{array}\right)
$$


Modulo the scalar factor $2 e^{i \theta}$, the unitary $\check{R}(\theta)$-matrix has the normalization factor $\rho$ by

$$
\rho=\sinh ^{2} \gamma+\sin ^{2} \theta .
$$

In addition, with the new variable $\theta$, such $\check{R}(\theta)$-matrix satisfies the following QYBE:

$$
\check{R}_{12}\left(\theta_{1}\right) \check{R}_{23}\left(\theta_{1}+\theta_{2}\right) \check{R}_{12}\left(\theta_{2}\right)=\check{R}_{23}\left(\theta_{1}\right) \check{R}_{12}\left(\theta_{1}+\theta_{2}\right) \check{R}_{23}\left(\theta_{2}\right) .
$$

Similarly, we can treat the case of $q=-e^{\gamma}$.

Let consider the BGR $b$-matrix which satisfies the Temperley-Lieb algebra and plays the role in constructing the Jones polynomial, see 4],

$$
b=\left(\begin{array}{cccc}
q & 0 & 0 & 0 \\
0 & 0 & 1 & 0 \\
0 & 1 & q-q^{-1} & 0 \\
0 & 0 & 0 & q
\end{array}\right)
$$

It has two eigenvalues: $q$ and $-q^{-1}$. With Yang-Baxterization, the corresponding $\check{R}(x)$-matrix has the form

$$
\check{R}(x)=\left(\begin{array}{cccc}
q-q^{-1} x & 0 & 0 & 0 \\
0 & \left(q-q^{-1}\right) x & 1-x & 0 \\
0 & 1-x & q-q^{-1} & 0 \\
0 & 0 & 0 & q-q^{-1} x
\end{array}\right) .
$$

Similar to the preceding case for the Alexander matrix, the unitarity condition (15) informs us that the spectral parameter $x$ lives on the unit circle $\|x\|=1$ and the deformation parameter $q$ has to be real.

We apply the ordinary unitarity requirements (15) instead of the unitarity conditions $\check{R}\left(x^{-1}\right) \check{R}(x) \propto \mathbb{1}$ often mentioned in literature related to the QYBE (8). With the latter one, we have the following the normalization factor $\rho$ by

$$
\rho=\check{R}(x) \check{R}\left(x^{-1}\right)=\left(q^{2}+q^{-2}-x-x^{-1}\right)
$$

for the non-standard representation and standard representation of the six-vertex model. It is compatible with the normalization factor (25).

\section{Unitary $\check{R}(x)$-matrix: the eight-vertex model}

In this section, we will present the complete solutions of the BGR (7) for the non-vanishing eight-vertex model and the corresponding unitary $\check{R}(x)$-matrices via Yang-Baxterization. In terms of non-vanishing Boltzman weights $w_{1}, w_{2}, \cdots$, $w_{8}$, the BGR $b$-matrix of the eight-vertex model assumes the form

$$
b=\left(\begin{array}{cccc}
w_{1} & 0 & 0 & w_{7} \\
0 & w_{5} & w_{3} & 0 \\
0 & w_{4} & w_{6} & 0 \\
w_{8} & 0 & 0 & w_{2}
\end{array}\right)
$$


Choosing suitable Boltzman weights leads to unitary solutions of the Yang-Baxter equation or the braid relation (7). In Appendix A.2, Yang-Baxterization used here is sketched in a practical way.

\subsection{Solutions of the eight-vertex model for $w_{3}=-w_{4}$ (I)}

Setting $w_{5}=w_{1}=w_{2}=w_{6}$ gives us $w_{1}^{2}=w_{3}^{2}=w_{4}^{2}$ and $w_{3}^{2}+w_{7} w_{8}=0$, see Appendix A.2. In the case of $w_{3} \neq w_{4}$, we have $w_{3}=-w_{4}$ and $w_{1}= \pm w_{3}$. The BGR $b$-matrix has the form

$$
b_{ \pm}=\left(\begin{array}{cccc}
w_{1} & 0 & 0 & w_{7} \\
0 & w_{1} & \pm w_{1} & 0 \\
0 & \mp w_{1} & w_{1} & 0 \\
-\frac{w_{1}^{2}}{w_{7}} & 0 & 0 & w_{1}
\end{array}\right) \Longleftrightarrow\left(\begin{array}{cccc}
1 & 0 & 0 & q \\
0 & 1 & \pm 1 & 0 \\
0 & \mp 1 & 1 & 0 \\
-q^{-1} & 0 & 0 & 1
\end{array}\right) .
$$

It has two eigenvalues $\lambda_{1}=1-i$ and $\lambda_{2}=1+i$. The corresponding $\check{R}(x)$ matrix via Yang-Baxterization is obtained to be

$$
\begin{aligned}
\check{R}_{ \pm}(x)= & b_{ \pm}+x \lambda_{1} \lambda_{2} b_{ \pm}^{-1} \\
= & \left(\begin{array}{cccc}
1+x & 0 & 0 & q(1-x) \\
0 & 1+x & \pm(1-x) & 0 \\
0 & \mp(1-x) & 1+x & 0 \\
-q^{-1}(1-x) & 0 & 0 & 1+x
\end{array}\right) .
\end{aligned}
$$

Assume the spectral parameter $x$ and the deformation parameter $q$ to be complex numbers. The unitarity condition (15) leads to the following equations

$$
\left\{\begin{array}{ccc}
\|1+x\|^{2}+\|q\|^{2}\|1-x\|^{2} & = & \rho \\
\|1+x\|^{2}+\frac{1}{\|q\|^{2}}\|1-x\|^{2} & = & \rho \\
\|1+x\|^{2}+\|1-x\|^{2} & = & \rho \\
(1-x)(1+\bar{x})-(1+x)(1-\bar{x}) & = & 0 \\
-q^{-1}(1-x)(1+\bar{x})+\bar{q}(1+x)(1-\bar{x}) & = & 0
\end{array}\right.
$$

which specify $x$ real and $q$ living at a unit circle.

Introducing the new variables of angles $\theta$ and $\varphi$ as follows

$$
\cos \theta=\frac{1}{\sqrt{1+x^{2}}}, \quad \sin \theta=\frac{x}{\sqrt{1+x^{2}}}, \quad q=e^{-i \varphi},
$$

we represent the $\check{R}_{ \pm}(x)$-matrix in a new form

$$
\check{R}_{ \pm}(\theta)=\cos \theta b_{ \pm}(\varphi)+\sin \theta\left(b_{ \pm}\right)^{-1}(\varphi)
$$

in which the BGR $b_{ \pm}(\varphi)$-matrices are given by

$$
b_{ \pm}(\varphi)=\frac{1}{\sqrt{2}}\left(\begin{array}{cccc}
1 & 0 & 0 & e^{-i \varphi} \\
0 & 1 & \pm 1 & 0 \\
0 & \mp 1 & 1 & 0 \\
-e^{i \varphi} & 0 & 0 & 1
\end{array}\right)
$$




\subsection{Solutions of the eight-vertex model for $w_{3}=w_{4}$ (II)}

Imposing $w_{5}=w_{6}$ and $w_{3}=w_{4}$ in the given eight-vertex model. In the first case of $\frac{w_{1}}{w_{5}}=2-t$, set $z=\left(t^{2}-2 t+2\right)^{\frac{1}{2}}$. The BGR $b_{ \pm}$-matrices take the form

$$
b_{ \pm}=\left(\begin{array}{cccc}
2-t & 0 & 0 & q \\
0 & 1 & \pm z & 0 \\
0 & \pm z & 1 & 0 \\
q^{-1} & 0 & 0 & t
\end{array}\right)
$$

It has two distinct eigenvalues $1 \pm z$ and so via Yang-Baxterization the $\check{R}(x)$ matrices are obtained by

$$
\check{R}_{ \pm}(x)=\left(\begin{array}{cccc}
2-t(1-x) & 0 & 0 & q(1-x) \\
0 & 1+x & \pm z(1-x) & 0 \\
0 & \pm z(1-x) & 1+x & 0 \\
q^{-1}(1-x) & 0 & 0 & 2 x+t(1-x)
\end{array}\right) .
$$

For simplicity, assume $t$ real and then $z$ positive real. The unitarity condition (15) leads to the following equations,

$$
\left\{\begin{array}{cc}
\|(2-t)+t x\|^{2}+q \bar{q}\|1-x\|^{2} & =\rho \\
\bar{q}^{-1}((2-t)+t x)(1-\bar{x})+q(1-x)(t+(2-t) \bar{x}) & =0 \\
\|1+x\|^{2}+z^{2}\|1-x\|^{2} & =\rho \\
z(1+x)(1-\bar{x})+z(1-x)(1+\bar{x}) & =0 \\
q^{-1} \bar{q}^{-1}\|1-x\|^{2}+\|t+(2-t) x\|^{2} & =\rho
\end{array} .\right.
$$

Analyzing the fourth equation leads to $\|x\|^{2}=1$, while comparing the first equation and the third one gives $\|q\|^{2}=1$. They survive the remaining equations. The normalization factor $\rho$ is given by

$$
\rho=2\left(1+z^{2}\right)+\left(1-z^{2}\right)(x+\bar{x})=4+(t-1)^{2}(2-x-\bar{x}) .
$$

\subsection{Solutions of the eight-vertex model for $w_{3}=w_{4}$ (III)}

Following the subsection (4.2), consider the second case of $w_{3}=w_{4}$, namely take $\frac{w_{1}}{w_{5}}=t$ so that $z= \pm t$. The corresponding BGR $b$-matrix has the form

$$
b_{ \pm}=\left(\begin{array}{cccc}
t & 0 & 0 & q \\
0 & 1 & \pm t & 0 \\
0 & \pm t & 1 & 0 \\
q^{-1} & 0 & 0 & t
\end{array}\right)
$$

which has three distinct eigenvalues. It needs two types of Yang-Baxterization. The first case is essentially the same as the case of two distinct eigenvalues, while the second case is treated in detail in Appendix A.2. 
With Yang-Baxterization of the first case, we have

$$
\check{R}_{ \pm}(x)=\left(\begin{array}{cccc}
t(1-x) & 0 & 0 & q(1+x) \\
0 & 1+x & \pm t(1-x) & 0 \\
0 & \pm t(1-x) & 1+x & 0 \\
q^{-1}(1+x) & 0 & 0 & t(1-x)
\end{array}\right) .
$$

The unitarity condition (15) shows

$$
\left\{\begin{array}{ccc}
\|t\|^{2}\|1-x\|^{2}+q \bar{q}\|1+x\|^{2} & = & \rho \\
\|t\|^{2}\|1-x\|^{2}+\|1+x\|^{2} & = & \rho \\
\|t\|^{2}\|1-x\|^{2}+q^{-1} \bar{q}^{-1}\|1+x\|^{2} & = & \rho \\
t(1-x)(1+\bar{x})+\bar{t}(1+x)(1-\bar{x}) & = & 0 \\
\bar{q}^{-1} t(1-x)(1+\bar{x})+q \bar{t}(1+x)(1-\bar{x}) & = & 0
\end{array} .\right.
$$

The first three equations give $\|q\|^{2}=1$. Simplifying the fourth equation gives

$$
\|x\|^{2}=1+\frac{1-t_{1}^{2}}{1+t_{1}^{2}}(x-\bar{x})
$$

where $t_{1}^{2}=\frac{t^{2}}{\|t\|^{2}}$.

Setting $t=\|t\| e^{i \phi}$ leads to

$$
\|x\|^{2}=1-i \tan \phi(x-\bar{x})
$$

and setting $x=a+i b$ gives us

$$
a^{2}+(b-\tan \phi)^{2}=\sec ^{2} \phi
$$

so that the normalization factor $\rho$ has the form

$$
\rho=2\left(1+\|t\|^{2}\right)-i \tan \phi(x-\bar{x})\left(1+\|t\|^{2}\right)+\left(1-\|t\|^{2}\right)(x+\bar{x}) .
$$

Assume $t$ real, namely $\phi=0$, then $\|x\|^{2}=1$ and the normalization factor $\rho$ is given by

$$
\rho=t^{2}(2-x-\bar{x})+2+x+\bar{x}
$$

which is non-negative since $\|x\|=1$.

\subsection{Solutions of the eight-vertex model for $w_{3}=w_{4}$ (IV)}

Following the subsection (4.3), the second case for the BGR $b$-matrix (41) having three distinct eigenvalues gives the following $\check{R}(x)$-matrix:

$$
\begin{aligned}
\check{R}_{ \pm}(x) & =\left(\begin{array}{cccc}
t(1+x) g_{1} & 0 & 0 & q(1-x) g_{1} \\
0 & (1+x) g_{2} & \pm t(1-x) g_{2} & 0 \\
0 & \pm t(1-x) g_{2} & (1+x) g_{2} & 0 \\
q^{-1}(1-x) g_{1} & 0 & 0 & t(1+x) g_{1}
\end{array}\right), \\
& \Longleftrightarrow\left(\begin{array}{cccc}
t(1+x) & 0 & 0 & q(1-x) \\
0 & (1+x) g & \pm t(1-x) g & 0 \\
0 & \pm t(1-x) g & (1+x) g & 0 \\
q^{-1}(1-x) & 0 & 0 & t(1+x)
\end{array}\right)
\end{aligned}
$$


where the symbols $g_{1}, g_{2}$ and $g$ are respectively defined by

$$
g_{1}=1+t+x(1-t), \quad g_{2}=1+t-x(1-t), \quad g=\frac{g_{2}}{g_{1}} .
$$

The unitarity condition (15) delivers us the following equations

$$
\left\{\begin{array}{ccc}
\|t\|^{2}\|1+x\|^{2}+\|q\|^{2}\|1-x\|^{2} & = & \rho \\
t \bar{q}^{-1}(1+x)(1-\bar{x})+\bar{t} q(1-x)(1+\bar{x}) & = & 0 \\
q^{-1} \bar{q}^{-1}\|1-x\|^{2}+\|t\|^{2}\|1+x\|^{2} & = & \rho \\
\|g\|^{2}\|1+x\|^{2}+\|t\|^{2}\|g\|^{2}\|1-x\|^{2} & = & \rho \\
\bar{t}\|g\|^{2}(1+x)(1-\bar{x})+t\|g\|^{2}(1+\bar{x})(1-x) & = & 0
\end{array} .\right.
$$

Identifying the first equation with the third one requires $\|q\|^{2}=1$. Identifying the second equation with the last one shows

$$
(t-\bar{t})(x-\bar{x})=0 \text {. }
$$

Focus on the second equation: as $t$ is real, we have $\|x\|=1$; as $x$ is real, we have $t=-\bar{t}$.

Comparing the first equation with the fourth one leads to the normalization factor $\rho$. For real $t$, it takes

$$
\rho=\left\|g_{2}\right\|^{2}=2\left(1+t^{2}\right)-\left(1-t^{2}\right)(x+\bar{x}) ;
$$

for pure imaginary $t$, it gives

$$
\rho=\left\|g_{2}\right\|^{2}=(1-x)^{2}+\|t\|^{2}(1+x)^{2} .
$$

\subsection{Two types of unitarity conditions}

In order to obtain the time-evolution of quantum entangled state determined by the unitary $\check{R}(x)$-matrix, it is better to consider the ordinary unitarity condition (15) instead of the unitarity condition $\check{R}(x) \check{R}\left(x^{-1}\right) \propto \mathbb{1}$.

For the first type of the eight-vertex model (32), we have

$$
\check{R}(x) \check{R}\left(x^{-1}\right)=2\left(x+x^{-1}\right) \mathbb{1},
$$

which is incompatible with the normalization factor $\rho$ by

$$
\rho=R(x) \check{R}^{\dagger}(\bar{x})=2\left(1+x^{2}\right) \mathbb{1}
$$

except $x=1$. For the second type of the eight-vertex model (38), we have

$$
\check{R}(x) \check{R}\left(x^{-1}\right)=2\left(1+z^{2}\right)+\left(1-z^{2}\right)\left(x+x^{-1}\right)
$$

which is compatible with the normalization factor (40) for real $t$. For the third type of the eight-vertex model (42), we have

$$
\check{R}(x) \check{R}\left(x^{-1}\right)=2\left(1+t^{2}\right)+\left(1-t^{2}\right)\left(x+x^{-1}\right)
$$

which is obviously an special example of the normalization factor (47). For the fourth type of the eight-vertex model (49), we have

$$
\check{R}(x) \check{R}\left(x^{-1}\right)=2\left(1+t^{2}\right)+\left(t^{2}-1\right)\left(x+x^{-1}\right)
$$

which is the same as the normalization factor (53) but not (54). 


\section{The $\check{R}(x)$-matrices as universal quantum gates}

In this section, we will view unitary $\check{R}(x)$-matrices as universal quantum gates with the help of the Brylinski's theorem 13. It is a natural generalization of the argument regarding a unitary braiding operator as a universal quantum gate. Therefore quantum entanglements not only see topological entanglements or topological invariants but also know geometric information or geometric invariants hidden in unitary solutions of the QYBE (8).

A pure state $|\psi\rangle$ denoted by

$$
|\psi\rangle=\sum_{i, j=0}^{1} a_{i j}|i j\rangle, \quad|i j\rangle=|i\rangle \otimes|j\rangle
$$

is entangled if $a_{00} a_{11} \neq a_{01} a_{10}$. The entanglement of $|\psi\rangle$ is equivalent to the statement that $|\psi\rangle$ is not the tensor product of two one-qubit states [39].

The $\check{R}$-matrix having the form

$$
\check{R}=\left(\begin{array}{cccc}
\check{R}_{00}^{00} & \check{R}_{01}^{00} & \check{R}_{10}^{00} & \check{R}_{11}^{00} \\
\check{R}_{00}^{01} & \check{R}_{01}^{01} & \check{R}_{10}^{01} & \check{R}_{11}^{01} \\
\check{R}_{00}^{10} & \check{R}_{01}^{10} & \check{R}_{10}^{10} & \check{R}_{11}^{10} \\
\check{R}_{00}^{11} & \check{R}_{01}^{11} & \check{R}_{10}^{11} & \check{R}_{11}^{11}
\end{array}\right),
$$

acts on the tensor product $|i\rangle \otimes|j\rangle$ via the formula

$$
\check{R}|i j\rangle=\sum_{k=0}^{1} \sum_{l=0}^{1} \check{R}_{i j}^{k l}|k l\rangle
$$

where $i, j, k, l$ take either 0 or 1 . The Brylinski's theorem [13] says that it is a universal quantum gate when it is a quantum entangling operator which transforms the tensor product $\left|\psi_{t p}\right\rangle$ into an entangling state $\check{R}\left|\psi_{t p}\right\rangle$ given by

$$
\check{R}\left|\psi_{p t}\right\rangle=\sum_{i, j=0}^{1} \sum_{k, l=0}^{1} \check{R}_{i j}^{k l} a_{i j}|k l\rangle=\sum_{k, l=0}^{1} b_{k l}|k l\rangle
$$

where the coefficients $a_{i j}$ satisfy $a_{00} a_{11}=a_{01} a_{10}$ and the coefficients $b_{k l}$ are defined by

$$
b_{k l}=\sum_{i, j=0}^{1} \check{R}_{i j}^{k l} a_{i j}
$$

satisfying $b_{00} b_{11} \neq b_{01} b_{10}$.

Introduce the four dimensional vectors $\vec{a}, \vec{b}$ and $\vec{r}_{i j}$ as

$$
\vec{a}=\left(\begin{array}{c}
a_{00} \\
a_{01} \\
a_{10} \\
a_{11}
\end{array}\right), \quad \vec{b}=\left(\begin{array}{c}
b_{00} \\
b_{01} \\
b_{10} \\
b_{11}
\end{array}\right), \quad \vec{r}_{i j}^{T}=\left(\begin{array}{c}
\check{R}_{00}^{i j} \\
\check{R}_{01}^{i j} \\
\check{R}_{10}^{i j} \\
\check{R}_{11}^{i j}
\end{array}\right)
$$


where the upper index $T$ denotes the transpose of the vector $\vec{r}_{i j}$, so that

$$
\check{R} \vec{a}=\vec{b}, \quad b_{i j}=\vec{r}_{i j} \cdot \vec{a} .
$$

For convenience of the following discussion, consider the $2 \times 2$ matrix $A$ instead of the vector $\vec{a}$ and the $2 \times 2$ matrix $B$ instead of the vector $\vec{b}$ as follows

$$
A=\left(\begin{array}{ll}
a_{00} & a_{01} \\
a_{10} & a_{11}
\end{array}\right), \quad B=\left(\begin{array}{cc}
b_{00} & b_{01} \\
b_{10} & b_{11}
\end{array}\right)
$$

The criteria of quantum entanglement for the quantum state determined by the matrix $A(B)$ is that the determinant of the matrix $A(B)$ is not zero, namely,

$$
\operatorname{Det}(A)=a_{00} a_{11}-a_{01} a_{10} \neq 0, \quad \operatorname{Det}(B)=b_{00} b_{11}-b_{01} b_{10} \neq 0
$$

where the determinant $\operatorname{Det}(A)$ (or $\operatorname{Det}(B))$ can be called as the concurrence of the corresponding quantum state, see [40, 41]. Here in order to judge whether the unitary $\check{R}(x)$-matrix is a universal quantum gate with the Brylinski's theorem 13], we choose $\operatorname{Det}(A)=0$ (so that the initial state is unentangled).

\subsection{The case for solutions of the six-vertex models}

For the non-standard representation (24) of the six-vertex model, the vector $\vec{b}$ is obtained to be

$$
\left(\begin{array}{l}
b_{00} \\
b_{01} \\
b_{10} \\
b_{11}
\end{array}\right)=\left(\begin{array}{l}
\sinh (\gamma-i \theta) a_{00} \\
e^{i \theta} \sinh \gamma a_{01}-i \sin \theta a_{10} \\
-i \sin \theta a_{01}+e^{-i \theta} \sinh \gamma a_{10} \\
\sinh (\gamma+i \theta) a_{11}
\end{array}\right)
$$

which gives

$$
\begin{aligned}
& b_{00} b_{11}=\left(\sinh ^{2} \gamma+\sin ^{2} \theta\right) a_{00} a_{11}, \\
& b_{01} b_{10}=\left(\sinh ^{2} \gamma-\sin ^{2} \theta\right) a_{01} a_{10}-i \sin \theta \sinh \gamma\left(a_{01}^{2} e^{i \theta}+a_{10}^{2} e^{-i \theta}\right) .
\end{aligned}
$$

So the criteria of quantum entanglement has the form

$$
\operatorname{Det}(B)=\sin \theta\left[2 a_{00} a_{11} \sin \theta+i\left(a_{01}^{2} e^{i \theta}+a_{10}^{2} e^{-i \theta}\right) \sinh \gamma\right] \neq 0 .
$$

Consider the case of the spectral parameter $x \neq 1$, namely $\sin \theta \neq 0$. The choice: $a_{00}=a_{10}=0, a_{01} \neq 0$ and $\gamma \neq 0$ satisfies $\operatorname{Det}(B) \neq 0$. When $\gamma=0$, namely the deformation parameter $q=1$, the criteria of quantum entanglement requires $a_{00} a_{11} \neq 0$. Hence the unitary $\check{R}(\theta)$-matrix for the non-standard representation of the six-vertex model is a universal quantum gate except for $x=1$.

For the standard representation (28) of the six-vertex model, the determinant of the matrix $B$ is a difference between two terms $b_{00} b_{11}$ and $b_{01} b_{10}$ given by

$$
\begin{aligned}
& b_{00} b_{11}=\sinh (\gamma-i \theta) \sinh (\gamma-i \theta) a_{00} a_{11} \\
& b_{01} b_{10}=\left(\sinh ^{2} \gamma-\sin ^{2} \theta\right) a_{01} a_{10}-i \sin \theta \sinh \gamma\left(a_{01}^{2} e^{i \theta}+a_{10}^{2} e^{-i \theta}\right)
\end{aligned}
$$


The choice: $a_{00}=a_{10}=0, a_{01} \neq 0$ and $\gamma \neq 0$ satisfies $\operatorname{Det}(B) \neq 0$. But the case of $\gamma=0$ leads to $\operatorname{Det}(B)=0$. Hence the unitary $\check{R}(\theta)$-matrix for the standard representation of the six-vertex model is a universal quantum gate except for $x=1$ or $q=1$.

To distinguish the non-standard representation from the standard representation in a clear way, the $\check{R}(x)$-matrices of $q=1$ are given respectively by

$$
\check{R}_{n o n}=\left(\begin{array}{cccc}
1 & 0 & 0 & 0 \\
0 & 0 & 1 & 0 \\
0 & 1 & 0 & 0 \\
0 & 0 & 0 & -1
\end{array}\right), \quad \check{R}_{\text {standard }}=\left(\begin{array}{cccc}
1 & 0 & 0 & 0 \\
0 & 0 & 1 & 0 \\
0 & 1 & 0 & 0 \\
0 & 0 & 0 & 1
\end{array}\right)
$$

in which the non-standard one for constructing the Alexander polynomial is a universal quantum gate and the standard one for constructing the Jones polynomial is not a universal quantum gate [2].

\subsection{The case for solutions of the eight-vertex model (I)}

For simplicity of analyzing the criteria of quantum entanglement, we introduce the new variable $u, v$ instead of the spectral parameter $x, y$ so that

$$
u=\frac{1-x}{1+x}, \quad v=\frac{1-y}{1+y}, \quad \frac{1-x y}{1+x y}=\frac{u+v}{1+u v}
$$

which suggest the following Yang-Baxter-like equation

$$
\check{R}_{12}(u) \check{R}_{23}\left(\frac{u+v}{1+u v}\right) \check{R}_{12}(v)=\check{R}_{23}(u) \check{R}_{12}\left(\frac{u+v}{1+u v}\right) \check{R}_{23}(v) .
$$

Specifying $x=e^{i \theta_{1}}$ and $y=e^{i \theta_{2}}$, the parameters in the above equation have the forms

$$
u=-i \tan \theta_{1}, v=-i \tan \theta_{2}, \frac{u+v}{1+u v}=-i \tan \left(\theta_{1}+\theta_{2}\right) .
$$

The unitary $\check{R}(u)$-matrix being a universal quantum gate suggests that the unitary $\check{R}(x)$-matrix is a universal quantum gate since the determinant $\operatorname{Det}(B)$ are not vanishing in both cases. In terms of the new variable $u$, the unitary $\check{R}_{ \pm}(u)$-matrix for the first type of solution (32) of the eight-vertex model has the form

$$
\check{R}_{ \pm}(u)=\left(\begin{array}{cccc}
1 & 0 & 0 & q u \\
0 & 1 & \pm u & 0 \\
0 & \mp u & 1 & 0 \\
-q^{-1} u & 0 & 0 & 1
\end{array}\right)
$$

The corresponding matrix $B^{ \pm}$is given by

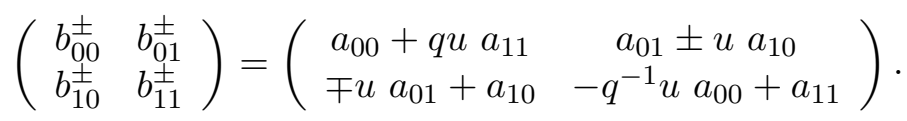


It has the determinant

$$
\operatorname{Det}\left(B^{ \pm}\right)=u\left(q a_{11}^{2}-q^{-1} a_{00}^{2} \pm a_{01}^{2} \mp a_{10}^{2}\right)
$$

which is not zero for the case of $u \neq 0(x \neq 1), a_{00}=a_{01}=0$ and $a_{10}^{2} \neq q a_{11}^{2}$. Hence the unitary $\check{R}_{ \pm}(u)$-matrix is a universal quantum gate except $u=0$, that is to say the unitary $\check{R}_{ \pm}(x)$-matrix is a universal quantum gate except $x=1$.

In terms of the coefficients $a, b, c, d$ given by

$$
a_{00}=a c, a_{01}=a d, a_{10}=b c, a_{11}=b d,
$$

instead of $a_{i j}$, the criteria of quantum entanglement leads to

$$
\left(d^{2} \mp q^{-1} c^{2}\right)\left(q b^{2} \pm a^{2}\right) \neq 0 .
$$

Consider which type of quantum states violate the criteria of quantum entanglements. For the $\check{R}_{+}$-matrix, we have

$$
d^{2}=q^{-1} c^{2}, \quad \text { or } \quad a^{2}=-q b^{2},
$$

while for the $\check{R}_{-}$-matrix, we have

$$
d^{2}=-q^{-1} c^{2}, \quad \text { or } \quad a^{2}=q b^{2} .
$$

Therefore, although a universal quantum gate is identified as an entangling operator, it is also able to transform a specified unentangled state (a tensor product of quantum states) into another unentangled state.

\subsection{The case for solutions of the eight-vertex model (II)}

In terms of the new variable $u$, the corresponding $\check{R}_{ \pm}(u)$-matrix has the form

$$
\check{R}_{ \pm}(u)=\left(\begin{array}{cccc}
1+(1-t) u & 0 & 0 & q u \\
0 & 1 & \pm z u & 0 \\
0 & \pm z u & 1 & 0 \\
q^{-1} u & 0 & 0 & 1+(t-1) u
\end{array}\right)
$$

so that the vector $\vec{b}^{ \pm}$are given by

$$
\left(\begin{array}{c}
b_{00}^{ \pm} \\
b_{01}^{ \pm} \\
b_{10}^{ \pm} \\
b_{11}^{ \pm}
\end{array}\right)=\left(\begin{array}{c}
(1+(1-t) u) a_{00}+q u a_{11} \\
a_{01} \pm z u a_{10} \\
\pm z u a_{01}+a_{10} \\
q^{-1} u a_{00}+(1+(t-1) u) a_{11}
\end{array}\right)
$$

which leads to

$$
\begin{aligned}
b_{00}^{ \pm} b_{11}^{ \pm}= & \left(1+\left(2-z^{2}\right) u^{2}\right) a_{00} a_{11} \\
& +u\left(q^{-1}(1+(1-t) u) a_{00}^{2}+q(1+(t-1) u) a_{11}^{2}\right), \\
b_{01}^{ \pm} b_{10}^{ \pm}= & \left(1+z^{2} u^{2}\right) a_{01} a_{10} \pm u z\left(a_{01}^{2}+a_{10}^{2}\right) .
\end{aligned}
$$

To satisfy $\operatorname{Det}(B) \neq 0$, choose $u \neq 0, a_{00}=a_{01}=a_{11}=0$ but $a_{10} \neq 0$. Hence the unitary $\check{R}_{ \pm}(x)$-matrix ([38) is a universal quantum gate for real $t$ and $x \neq 1$. 


\subsection{The case for solutions of the eight-vertex model (III)}

With the new variable $u$, the $\check{R}_{ \pm}(u)$-matrix is given by

$$
\check{R}_{ \pm}(u)=\left(\begin{array}{cccc}
t u & 0 & 0 & q \\
0 & 1 & \pm t u & 0 \\
0 & \pm t u & 1 & 0 \\
q^{-1} & 0 & 0 & t u
\end{array}\right)
$$

which says

$$
\begin{aligned}
& b_{00}^{ \pm} b_{11}^{ \pm}=\left(1+t^{2} u^{2}\right) a_{00} a_{11}+u t\left(q a_{11}^{2}+q^{-1} a_{00}^{2}\right) \\
& b_{01}^{ \pm} b_{10}^{ \pm}=\left(1+t^{2} u^{2}\right) a_{00} a_{11} \pm u t\left(a_{01}^{2}+a_{10}^{2}\right)
\end{aligned}
$$

So the unitary $\check{R}(x)$-matrix (42) is a universal quantum gate except $t u=0$.

The criteria of quantum entanglement in terms of the coefficients (80) has the form

$$
\left(a^{2} \mp b^{2} q^{-1}\right)\left(c^{2} q \mp d^{2}\right) \neq 0 .
$$

For the unitary $\check{R}_{ \pm}(x)$-matrix, the quantum state specified by

$$
a^{2}= \pm b^{2} q^{-1}, \quad \text { or } \quad d^{2}= \pm c^{2} q
$$

is not entangling even under the action of the unitary $\check{R}_{ \pm}(x)$-matrix.

\subsection{The case for solutions of the eight-vertex model (IV)}

With the new variable $u$, the $\check{R}_{ \pm}(u)$-matrix has the form

$$
\check{R}_{ \pm}(u)=\left(\begin{array}{cccc}
t(1+t u) & 0 & 0 & q u(1+t u) \\
0 & u+t & \pm t u(u+t) & 0 \\
0 & \pm t u(u+t) & u+t & 0 \\
q^{-1} u(1+t u) & 0 & 0 & t(1+t u)
\end{array}\right)
$$

which gives us

$$
\begin{aligned}
& b_{00}^{ \pm} b_{11}^{ \pm}=\left(t^{2}+u^{2}\right)(1+t u)^{2} a_{00} a_{11}+u t(1+t u)^{2}\left(q^{-1} a_{00}^{2}+q a_{11}^{2}\right), \\
& b_{01}^{ \pm} b_{10}^{ \pm}=\left(1+t^{2} u^{2}\right)(u+t)^{2} a_{00} a_{11} \pm u t(u+t)^{2}\left(a_{01}^{2}+a_{10}^{2}\right) .
\end{aligned}
$$

It can be observed that the unitary $\check{R}_{ \pm}(x)$-matrix (49) is a universal quantum gate for $u t \neq 0$.

\section{The constructions of the Hamiltonian}

In this section, we present a method of constructing the Hamiltonian from the unitary $\check{R}(x)$-matrix $(\check{R}(\theta)$-matrix) for the six-vertex and eight-vertex model. The comments on our construction are given in the last subsection. 
The wave function $\psi(x)$ is specified by the unitary $\check{R}(x)$-matrix, with $\psi(x)=$ $\check{R}(x) \psi$, the pure state $\psi$ independent of the time (or the spectral parameter $x$ ). Hence we obtain the Shrödinger equation corresponding to the time evolution of $\psi(x)$ controlled by the unitary $\check{R}(x)$-matrix,

$$
i \frac{\partial \psi(x)}{\partial x}=H(x) \psi(x), \quad H(x)=i \frac{\partial \check{R}(x)}{\partial x} \check{R}^{-1}(x)
$$

Here the unitary $\check{R}(x)$-matrix has to be the form containing the normalization factor, namely $\rho^{-\frac{1}{2}} \check{R}(x)$ satisfying $\left(\rho^{-\frac{1}{2}} \check{R}\right)^{-1}=\left(\rho^{-\frac{1}{2}} \check{R}\right)^{\dagger}$. The "time-dependent" Hamiltonian $H(x)$ is obtained to be

$$
H(x)=i \frac{\partial\left(\rho^{-\frac{1}{2}} \check{R}\right)}{\partial x}\left(\rho^{-\frac{1}{2}} \check{R}\right)^{-1}(x)=i \frac{\partial\left(\rho^{-\frac{1}{2}} \check{R}\right)}{\partial x} \rho^{-\frac{1}{2}} \check{R}^{\dagger}(x) .
$$

It is expanded into the form

$$
\begin{aligned}
H(x) & =i\left(\partial_{x} \ln \rho^{-\frac{1}{2}}(x)+\partial_{x} \check{R}(x) \check{R}^{-1}(x)\right) \\
& =i \rho^{-1}(x)\left(-\frac{1}{2} \frac{\partial \rho(x)}{\partial x}+\frac{\partial \check{R}(x)}{\partial x} \check{R}^{\dagger}(x)\right)
\end{aligned}
$$

where the formula in terms of $\check{R}^{\dagger}(x)$ is applied in the following since the calculation of $\check{R}^{\dagger}(x)$ is easier than that of $\check{R}^{-1}(x)$.

In this paper, the spectral parameter $x$ often takes $\|x\|=1$, namely $x=e^{i \theta}$ so the Hamiltonian $H(\theta)$ has another form

$$
H(\theta)=i \rho^{-1}(\theta)\left(-\frac{1}{2} \frac{\partial \rho(\theta)}{\partial \theta}+\frac{\partial \check{R}(\theta)}{\partial \theta} \check{R}^{\dagger}(\theta)\right)
$$

which leads to the following Schrödinger equation

$$
i \frac{\partial \psi(\theta)}{\partial \theta}=H(\theta) \psi(\theta)
$$

\subsection{The case for solutions of the six-vertex models}

For the non-standard representation (24) of the six-vertex model, the Hamiltonian $H(\theta)$ is constructed as follows

$$
H(\theta)=\frac{\sinh \gamma}{\rho}\left(\begin{array}{cccc}
\operatorname{coth} \gamma & 0 & 0 & 0 \\
0 & -\sinh \gamma & 1 & 0 \\
0 & 1 & \sinh \gamma & 0 \\
0 & 0 & 0 & -\operatorname{coth} \gamma
\end{array}\right)
$$

where the following formulas are used in calculation

$$
\begin{aligned}
& \sinh (a+b)=\sinh a \operatorname{coth} b+\sinh b \operatorname{coth} a, \quad \sinh i a=i \sin a \\
& \operatorname{coth}(a+b)=\operatorname{coth} a \operatorname{coth} b+\sinh a \sinh b, \quad \operatorname{coth} i a=\cos a .
\end{aligned}
$$


Here the Pauli matrices $\sigma_{x}, \sigma_{y}$ and $\sigma_{z}$ are set up as usual

$$
\sigma_{x}=\left(\begin{array}{cc}
0 & 1 \\
1 & 0
\end{array}\right), \quad \sigma_{y}=\left(\begin{array}{cc}
0 & -i \\
i & 0
\end{array}\right), \quad \sigma_{z}=\left(\begin{array}{cc}
1 & 0 \\
0 & -1
\end{array}\right) .
$$

In addition, the new matrices $\sigma_{ \pm}$are introduced by $\sigma_{ \pm}=\frac{1}{2}\left(\sigma_{x} \pm i \sigma_{y}\right)$ :

$$
\sigma_{+}=\left(\begin{array}{cc}
0 & 1 \\
0 & 0
\end{array}\right), \quad \sigma_{-}=\left(\begin{array}{cc}
0 & 0 \\
0 & 1
\end{array}\right) .
$$

To represent the Hamiltonian (98) in terms of the Pauli matrices, the formulas like

$$
\frac{1}{2}\left(\mathbb{1} \otimes \sigma_{z}+\sigma_{z} \otimes \mathbb{1}\right)=\left(\begin{array}{cccc}
1 & 0 & 0 & 0 \\
0 & 0 & 0 & 0 \\
0 & 0 & 0 & 0 \\
0 & 0 & 0 & -1
\end{array}\right), \sigma_{+} \otimes \sigma_{-}=\left(\begin{array}{cccc}
0 & 0 & 0 & 0 \\
0 & 0 & 1 & 0 \\
0 & 0 & 0 & 0 \\
0 & 0 & 0 & 0
\end{array}\right)
$$

are often used.

The Hamiltonian (98) has another form by the Pauli matrices

$$
\begin{aligned}
H(\theta)= & \frac{\sinh \gamma}{2 \rho}\left[\operatorname{coth} \gamma\left(\mathbb{1} \otimes \sigma_{z}+\sigma_{z} \otimes \mathbb{1}\right)+\sinh \gamma\left(\mathbb{1} \otimes \sigma_{z}-\sigma_{z} \otimes \mathbb{1}\right)\right. \\
& \left.+\left(\sigma_{+} \otimes \sigma_{-}+\sigma_{-} \otimes \sigma_{+}\right)\right] \\
= & \frac{\sinh \gamma}{2 \rho}\left[\operatorname{coth} \gamma\left(\mathbb{1} \otimes \sigma_{z}+\sigma_{z} \otimes \mathbb{1}\right)+\left(\sigma_{x} \otimes \sigma_{x}+\sigma_{y} \otimes \sigma_{y}\right)\right. \\
& \left.+\sinh \gamma\left(\mathbb{1} \otimes \sigma_{z}-\sigma_{z} \otimes \mathbb{1}\right)\right]
\end{aligned}
$$

where only the normalization factor $\rho$ depends on the time variable $\theta$ by

$$
\rho=\sin ^{2} \theta+\sinh ^{2} \gamma .
$$

For the standard representation (28) of the six-vertex model, the Hamiltonian $H(\theta)$ is obtained to be

$$
H(\theta)=\frac{\sinh \gamma}{\rho}\left(\begin{array}{cccc}
\operatorname{coth} \gamma & 0 & 0 & 0 \\
0 & -\sinh \gamma & 1 & 0 \\
0 & 1 & \sinh \gamma & 0 \\
0 & 0 & 0 & \operatorname{coth} \gamma
\end{array}\right)
$$

In terms of the Pauli-matrices, it has the form

$$
\begin{aligned}
H(\theta)= & \frac{\sinh \gamma}{2 \rho}\left[\operatorname{coth} \gamma\left(\mathbb{1}+\sigma_{z} \otimes \sigma_{z}\right)+\sinh \gamma\left(\mathbb{1} \otimes \sigma_{z}-\sigma_{z} \otimes \mathbb{1}\right)\right. \\
& \left.+\left(\sigma_{+} \otimes \sigma_{-}+\sigma_{-} \otimes \sigma_{+}\right)\right] \\
= & \frac{\sinh \gamma}{2 \rho}\left[\operatorname{coth} \gamma \mathbb{1}+\sinh \gamma\left(\mathbb{1} \otimes \sigma_{z}-\sigma_{z} \otimes \mathbb{1}\right)\right. \\
& \left.+\left(\sigma_{x} \otimes \sigma_{x}+\sigma_{y} \otimes \sigma_{y}+\operatorname{coth} \gamma \sigma_{z} \otimes \sigma_{z}\right)\right]
\end{aligned}
$$

which is different from the Hamiltonian (103) for the non-standard representation of the six-vertex model. 


\subsection{The case for solutions of the eight-vertex model (I)}

With the unitary solution $\check{R}(x)$ (32) of the QYBE (8), we construct the timeindependent Hamiltonian $H_{ \pm}$having the form

$$
H_{ \pm}=\left.i \frac{\partial}{\partial x}\left(\rho^{-\frac{1}{2}} \check{R}_{ \pm}\right)\right|_{x=1}=-\frac{i}{2} b_{ \pm}^{2}=\frac{i}{2}\left(\begin{array}{cccc}
0 & 0 & 0 & -e^{-i \varphi} \\
0 & 0 & \mp 1 & 0 \\
0 & \pm 1 & 0 & 0 \\
e^{i \varphi} & 0 & 0 & 0
\end{array}\right)
$$

Interestingly, we have the "time-dependent" Hamiltonian $H_{ \pm}(x)$ by

$$
H_{ \pm}(x)=i \frac{\partial\left(\rho^{-\frac{1}{2}} \check{R}_{ \pm}\right)}{\partial x} \rho^{-\frac{1}{2}} \check{R}_{ \pm}^{\dagger}(x)=-\frac{i}{1+x^{2}} b_{ \pm}^{2}
$$

which derives the above Hamiltonian $H_{ \pm}$at $x=1$. When $x$ is real, the Hamiltonian $H_{ \pm}(x)$ is a Hermitian operator.

For simplicity, we study the unitary $\check{R}(\theta)$-matrix (35) to decide the unitary evolution of quantum states. After some algebra, the Hamiltonian $H_{ \pm}$is obtained to be

$$
H_{ \pm}(\theta)=i \rho^{-1}(\theta)\left(-\frac{1}{2} \frac{\partial \rho(\theta)}{\partial \theta}+\frac{\partial \check{R}_{ \pm}(\theta)}{\partial \theta} \check{R}_{ \pm}^{\dagger}(\theta)\right)=\frac{i}{2} \frac{\partial x}{\partial \theta} H_{ \pm}(x)=H_{ \pm}
$$

which is independent of the time variable $\theta$.

In terms of the Pauli matrices $\sigma_{x}, \sigma_{y}$ and $\sigma_{z}$ and $\sigma_{ \pm}$, the Hamiltonian (109) has the form

$$
H_{ \pm}=\frac{i}{2}\left(-e^{-i \varphi} \sigma_{+} \otimes \sigma_{+}+e^{i \varphi} \sigma_{-} \otimes \sigma_{-} \mp \sigma_{+} \otimes \sigma_{-} \pm \sigma_{-} \otimes \sigma_{+}\right) .
$$

Introducing the two-dimensional vector $\vec{\sigma}$ and two unit directional vector $\vec{n}_{1}$ and $\vec{n}_{2}$ in $x y$-plane:

$$
\vec{\sigma}=\left(\sigma_{x}, \sigma_{y}\right) ; \quad \vec{n}_{1}=\left(\cos \frac{\pi+\varphi}{2}, \sin \frac{\pi+\varphi}{2}\right), \vec{n}_{2}=\left(\cos \frac{\varphi}{2}, \sin \frac{\varphi}{2}\right),
$$

the projections of the vector $\vec{\sigma}$ into $\vec{n}_{1}$ and $\vec{n}_{2}$ are given by

$$
\begin{aligned}
& \sigma_{n_{1}}=\vec{\sigma} \cdot \vec{n}_{1}=\sigma_{+} e^{-\frac{i}{2}(\varphi+\pi)}+\sigma_{-} e^{\frac{i}{2}(\varphi+\pi)}, \\
& \sigma_{n_{2}}=\vec{\sigma} \cdot \vec{n}_{2}=\sigma_{+} e^{-\frac{i}{2} \varphi}+\sigma_{-} e^{\frac{i}{2} \varphi} .
\end{aligned}
$$

The Hamiltonian (110) can be recast to

$$
H_{+}=\frac{1}{2} \sigma_{n_{1}} \otimes \sigma_{n_{2}}, \quad H_{-}=\frac{1}{2} \sigma_{n_{2}} \otimes \sigma_{n_{1}} .
$$

Consider the time-evolution operator $U_{ \pm}(\theta)$ determined by the Hamiltonian $H_{ \pm}$, for example, $U_{+}(\theta)$ given by

$$
U_{+}(\theta)=e^{-\frac{i}{2}\left(\sigma_{n_{1}} \otimes \sigma_{n_{2}}\right) \theta}=\cos \frac{\theta}{2}-i \sin \frac{\theta}{2} \sigma_{n_{1}} \otimes \sigma_{n_{2}} .
$$




\subsection{The case for solutions of the eight-vertex model (II)}

The unitary $\check{R}_{ \pm}(x)$-matrix (38) requires $x=e^{i \theta},\|q\|^{2}=1$ and real $t$. The normalization factor $\rho$ and its derivative have the forms

$$
\rho=4+4(t-1)^{2} \sin ^{2} \frac{\theta}{2}, \quad \frac{\partial \rho}{\partial \theta}=2(t-1)^{2} \sin \theta .
$$

After some calculation, the Hamiltonian $H_{ \pm}(\theta)$ is obtained to be

$$
\begin{aligned}
H_{ \pm}(\theta) & =i \rho^{-1}(\theta)\left(-\frac{1}{2} \frac{\partial \rho(\theta)}{\partial \theta}+\frac{\partial \check{R}_{ \pm}(\theta)}{\partial \theta} \check{R}_{ \pm}^{\dagger}(\theta)\right) \\
& =-\frac{\mathbb{1}}{2}+2 \rho^{-1}\left(\begin{array}{cccc}
1-t & 0 & 0 & q \\
0 & 0 & \pm z & 0 \\
0 & \pm z & 0 & 0 \\
q^{-1} & 0 & 0 & t-1
\end{array}\right) .
\end{aligned}
$$

In terms of the Pauli matrices, it is shown up as follows

$$
\begin{aligned}
H_{ \pm}(\theta)= & -\frac{1}{2} \mathbb{1}+(1-t) \rho^{-1}\left(\mathbb{1} \otimes \sigma_{z}+\sigma_{z} \otimes \mathbb{1}\right) \\
& +\frac{2}{\rho}\left[q \sigma_{+} \otimes \sigma_{+}+q^{-1} \sigma_{-} \otimes \sigma_{-} \pm z\left(\sigma_{+} \otimes \sigma_{-}+\sigma_{-} \otimes \sigma_{+}\right)\right] .
\end{aligned}
$$

Consider two special cases of making the normalization factor $\rho$ independent of the time variable $\theta$. Take $\theta=0$, namely, choose the Hamiltonian $H_{ \pm}(\theta)$ defined as

$$
H_{ \pm}(\theta)=\left.i \frac{\partial}{\partial \theta}\left(\rho^{-\frac{1}{2}} \check{R}_{ \pm}(\theta)\right)\right|_{\theta=0}
$$

which leads to the time-independent Hamiltonian

$$
\begin{aligned}
\left.H_{ \pm}\right|_{\theta=0}= & \frac{1}{2}\left[q \sigma_{+} \otimes \sigma_{+}+q^{-1} \sigma_{-} \otimes \sigma_{-} \pm z\left(\sigma_{+} \otimes \sigma_{-}+\sigma_{-} \otimes \sigma_{+}\right)\right] \\
& -\frac{1}{2} \mathbb{1}+\frac{1-t}{4}\left(\mathbb{1} \otimes \sigma_{z}+\sigma_{z} \otimes \mathbb{1}\right) .
\end{aligned}
$$

Take the parameter $t=1$, the time-independent Hamiltonian $H_{ \pm}$is given by

$$
H_{ \pm}=\frac{1}{2}\left[-\mathbb{1}+q \sigma_{+} \otimes \sigma_{+}+q^{-1} \sigma_{-} \otimes \sigma_{-} \pm\left(\sigma_{+} \otimes \sigma_{-}+\sigma_{-} \otimes \sigma_{+}\right)\right] .
$$

With the two unit directional vector $\vec{n}_{1}$ and $\vec{n}_{2}$ in $x y$-plane:

$$
\vec{n}_{1}=\left(\cos \frac{\varphi}{2}, \sin \frac{\varphi}{2}\right), \vec{n}_{2}=\left(\cos \frac{\pi+\varphi}{2}, \sin \frac{\pi+\varphi}{2}\right), q=e^{-i \varphi}
$$

the Hamiltonians (120) have the following forms

$$
H_{+}=\frac{1}{2}\left(-\mathbb{1}+\sigma_{n_{1}} \otimes \sigma_{n_{1}}\right), \quad H_{-}=-\frac{1}{2}\left(\mathbb{1}+\sigma_{n_{2}} \otimes \sigma_{n_{2}}\right) .
$$

Consider the unitary time-evolution operator $U_{ \pm}(\theta)$, for example, $U_{+}(\theta)$ given by

$$
U_{+}(\theta)=e^{-i H_{+} \theta}=e^{\frac{i \theta}{2}}\left(\cos \frac{\theta}{2}-i \sin \frac{\theta}{2} \sigma_{n_{1}} \otimes \sigma_{n_{1}}\right) .
$$




\subsection{The case for solutions of the eight-vertex model (III)}

Choose real $t, x=e^{i \theta}$ and $\|q\|=1$ for the unitary $\check{R}_{ \pm}(x)$-matrix (42). The normalization factor $\rho$ and its derivative are given by

$$
\rho=4+4\left(t^{2}-1\right) \sin ^{2} \frac{\theta}{2}, \quad \frac{\partial \rho}{\partial \theta}=2\left(t^{2}-1\right) \sin \theta .
$$

The corresponding Hamiltonian $H_{ \pm}(\theta)$ is found to be

$$
H_{ \pm}(\theta)=-\frac{1}{2} \mathbb{1}+\frac{2 t}{\rho}\left(\begin{array}{cccc}
0 & 0 & 0 & q \\
0 & 0 & \pm 1 & 0 \\
0 & \pm 1 & 0 & 0 \\
q^{-1} & 0 & 0 & 0
\end{array}\right)
$$

which leads to the Hamiltonian represented by the Pauli matrices

$$
H_{ \pm}(\theta)=-\frac{1}{2} \mathbb{1}+\frac{2 t}{\rho}\left[q \sigma_{+} \otimes \sigma_{+}+q^{-1} \sigma_{-} \otimes \sigma_{-} \pm\left(\sigma_{+} \otimes \sigma_{-}+\sigma_{-} \otimes \sigma_{+}\right)\right] .
$$

In the case of $\theta=0$, the time-independent Hamiltonian is given by

$$
\left.H_{ \pm}\right|_{\theta=0}=-\frac{1}{2} \mathbb{1}+\frac{t}{2}\left[q \sigma_{+} \otimes \sigma_{+}+q^{-1} \sigma_{-} \otimes \sigma_{-} \pm\left(\sigma_{+} \otimes \sigma_{-}+\sigma_{-} \otimes \sigma_{+}\right)\right] .
$$

In the other case of $t=1$, the other time-independent Hamiltonian $H_{ \pm}$has the form the same as (120).

\subsection{The case for solutions of the eight-vertex model (IV)}

Consider $x=e^{i \theta},\|q\|=1$ and real $t$ for the unitary $\check{R}_{ \pm}(x)$-matrix (49). The factor $\left\|g_{1}\right\|^{2},\left\|g_{2}\right\|^{2}$ and the normalization factor $\rho$ take the forms

$$
\begin{aligned}
\left\|g_{1}\right\|^{2} & =2\left(1+t^{2}\right)+2\left(1-t^{2}\right) \cos \theta, \quad\left\|g_{2}\right\|^{2}=2\left(1+t^{2}\right)-2\left(1-t^{2}\right) \cos \theta \\
\rho & =\left\|g_{1}\right\|^{2}\left\|g_{2}\right\|^{2}=4\left(1+t^{2}\right)^{2}-4\left(1-t^{2}\right)^{2} \cos ^{2} \theta
\end{aligned}
$$

Through some calculation, the Hamiltonian $H_{ \pm}(\theta)$ is obtained to be

$$
H_{ \pm}(\theta)=-\mathbb{1}+\frac{2 t}{\rho}\left(\begin{array}{cccc}
\left\|g_{2}\right\|^{2} & 0 & 0 & q\left\|g_{1}\right\|^{2} \\
0 & \left\|g_{1}\right\|^{2} & \pm\left\|g_{2}\right\|^{2} & 0 \\
0 & \pm\left\|g_{2}\right\|^{2} & \left\|g_{1}\right\|^{2} & 0 \\
q^{-1}\left\|g_{1}\right\|^{2} & 0 & 0 & \left\|g_{2}\right\|^{2}
\end{array}\right)
$$

which gives the following Hamiltonian as

$$
\begin{array}{r}
H_{ \pm}(\theta)=-\mathbb{1}+\frac{2 t}{\rho}\left[\frac{1}{2}\left(\left\|g_{1}\right\|^{2}+\left\|g_{2}\right\|^{2}\right) \mathbb{1} \otimes \mathbb{1}+\frac{1}{2}\left(\left\|g_{2}\right\|^{2}-\left\|g_{1}\right\|^{2}\right) \sigma_{z} \otimes \sigma_{z}\right. \\
\left.+\left\|g_{1}\right\|^{2}\left(q \sigma_{+} \otimes \sigma_{+}+q^{-1} \sigma_{-} \otimes \sigma_{-}\right) \pm\left\|g_{2}\right\|^{2}\left(\sigma_{+} \otimes \sigma_{-}+\sigma_{-} \otimes \sigma_{+}\right)\right] .
\end{array}
$$


The time-independent Hamiltonian obtained by taking $\theta=0$ is given by

$$
\begin{array}{r}
\left.H_{ \pm}\right|_{\theta=0}=-\mathbb{1}+\frac{1}{4 t}\left[\left(t^{2}+1\right) \mathbb{1} \otimes \mathbb{1}+\left(t^{2}-1\right) \sigma_{z} \otimes \sigma_{z}\right. \\
\left.+2\left(q \sigma_{+} \otimes \sigma_{+}+q^{-1} \sigma_{-} \otimes \sigma_{-}\right) \pm 2 t^{2}\left(\sigma_{+} \otimes \sigma_{-}+\sigma_{-} \otimes \sigma_{+}\right)\right],
\end{array}
$$

while the other time-independent Hamiltonian $H_{ \pm}$obtained by taking $t=1$ is the same as (120).

\subsection{Comments on our constructions of Hamiltonian}

The Schrodinger equation is a differential equation. It's solution represents the evolution of the initial state (input). Here before treating the Schrodinger equation, the time evolution of the state (the unitary braiding operator) is known as a discrete evolution and is determined by the unitary $\check{R}(x)$-matrix. Our problem is to find out which type of the Schrodinger equation has evolutionary solutions the same as the evolutions given by the unitary $\check{R}(x)$-matrices. In our case, therefore, the Schrodinger evolution is recognized as the unitary $\check{R}(x)$-matrix.

However, the Schrodinger equation says more than just the unitary $\check{R}(x)$ matrix. It leads us to study the physics behind it, and gives us new unitary solutions which are not necessarily solutions to the QYBE. The construction of a Hamiltonian from the unitary $\check{R}(x)$-matrix shows that the QYBE is a physical subject. In history, the Schrodinger equation is the starting point and how to solve the Schrodinger equation is a central topic. Now we try to recover(relate) physics from (to) the unitary $\check{R}(x)$-matrix.

In our case, the construction of the Hamiltonian in terms of the unitary $\check{R}(x)$ matrix follows a traditional approach in literature on the QYBE. There are two ways of choosing the time-variable. Each choice is determined by a corresponding purpose. We explain the spectral parameter $x(\theta)$ as the time variable. That is to say that we choose the time-evolution of quantum state (the unitary braiding operator) as the unitary $\check{R}(x)$-matrix. This is a natural and necessary choice in the sense of regarding unitary $\check{R}$-matrices as universal quantum gates. We want to see the evolution of unitary braiding operator or quantum state.

Indeed in the literature, the ordinary time in space-time as the time variable, the spectral parameter can be explained as the momentum, but the time-evolution of quantum state determined by the Hamiltonian can not be identified with the unitary $\check{R}(x)$-matrix. The physics in the case is well-known such as XXX model (and so on).

Finally it seems that the Schrodinger equation in our construction does not have space variables like the ordinary Schrodinger equation has. We could explain that we have physics on lattices of space: discrete physics. It seems that physics in our case is close to that on spin chains like XXX model (and so on). 


\section{The CNOT gates via the $\check{R}$-matrix}

The gate $G$ is universal for quantum computation (or just universal) if $G$ together with local unitary transformations (unitary transformations from $V$ to $V$ ) generates all unitary transformations of the complex vector space of dimension $2^{n}$ to itself. It is well-known [1] that the CNOT gate is a universal gate.

In [10], Kauffman and Lomonaco prove the following result.

Theorem 1. Let

$$
\check{R}=\left(\begin{array}{cccc}
1 / \sqrt{2} & 0 & 0 & 1 / \sqrt{2} \\
0 & 1 / \sqrt{2} & -1 / \sqrt{2} & 0 \\
0 & 1 / \sqrt{2} & 1 / \sqrt{2} & 0 \\
-1 / \sqrt{2} & 0 & 0 & 1 / \sqrt{2}
\end{array}\right)
$$

be the above unitary solution to the braid relation (17). Then $\check{R}$ is a universal gate. The proof below (repeated from 10]) gives a specific expression for the CNOT gate in terms of $\check{R}$.

Proof. This result follows at once from the Brylinksis' theorem [13, since $\check{R}$ is highly entangling. For a direct computational proof, it suffices to show that the CNOT gate can be generated from $\check{R}$ and local unitary transformations. Let

$$
\begin{aligned}
\alpha=\left(\begin{array}{cc}
1 / \sqrt{2} & 1 / \sqrt{2} \\
1 / \sqrt{2} & -1 / \sqrt{2}
\end{array}\right), & \beta=\left(\begin{array}{cc}
-1 / \sqrt{2} & 1 / \sqrt{2} \\
i / \sqrt{2} & i / \sqrt{2}
\end{array}\right) \\
\gamma=\left(\begin{array}{cc}
1 / \sqrt{2} & i / \sqrt{2} \\
1 / \sqrt{2} & -i / \sqrt{2}
\end{array}\right), & \delta=\left(\begin{array}{cc}
1 & 0 \\
0 & i
\end{array}\right) .
\end{aligned}
$$

Let $M=\alpha \otimes \beta$ and $N=-\gamma \otimes \delta$. Then it is straightforward to verify that

$$
\mathrm{CNOT}=M \cdot \check{R} \cdot N \text {. }
$$

This completes the proof.

We now show how Yang-Baxterization illuminates the structure of this Bell basis transformation. We discuss physics related to the time-evolution of the universal quantum gate determined by the unitary $\check{R}(\theta)$-matrix (35). The braid group representation $b_{ \pm}(\varphi)$-matrix (36) yields the Bell states with the phase factor $e^{i \varphi}$,

$$
b_{ \pm}(\varphi)\left(\begin{array}{c}
|00\rangle \\
|01\rangle \\
|10\rangle \\
|11\rangle
\end{array}\right)=\frac{1}{\sqrt{2}}\left(\begin{array}{c}
|00\rangle-e^{i \varphi}|11\rangle \\
|01\rangle \mp|10\rangle \\
\pm|01\rangle+|10\rangle \\
e^{-i \varphi}|00\rangle+|11\rangle
\end{array}\right)
$$

which shows that $\varphi=0$ leads to the Bell states, the maximum of entangled states,

$$
\frac{1}{\sqrt{2}}(|00\rangle \pm|11\rangle), \quad \frac{1}{\sqrt{2}}(|10\rangle \pm|01\rangle) .
$$


In terms of the Hamiltonian $H_{ \pm}$(109), the $\check{R}_{ \pm}(\theta)$-matrix (35) has the form

$$
\check{R}_{ \pm}(\theta)=\cos \left(\frac{\pi}{4}-\theta\right)+2 i \sin \left(\frac{\pi}{4}-\theta\right) H_{ \pm}=e^{i\left(\frac{\pi}{2}-2 \theta\right) H_{ \pm}}
$$

which can be also used to construct the CNOT gate with additional single qubit transformations, examples see [10]. The unitary $\check{R}$-matrix (132) is realized by

$$
\check{R}=\left.\check{R}_{-}(\theta)\right|_{\theta=\varphi=0}=e^{i \frac{\pi}{4}\left(\sigma_{x} \otimes \sigma_{y}\right)} .
$$

In addition, with the unitary $\check{R}(\theta)$-matrix (35), we have

$$
\check{R}_{ \pm}(\theta)\left(\begin{array}{l}
|00\rangle \\
|01\rangle \\
|10\rangle \\
|11\rangle
\end{array}\right)=\left(\begin{array}{l}
\cos \left(\frac{\pi}{4}-\theta\right)|00\rangle-e^{i \varphi} \sin \left(\frac{\pi}{4}-\theta\right)|11\rangle \\
\cos \left(\frac{\pi}{4}-\theta\right)|01\rangle \mp \sin \left(\frac{\pi}{4}-\theta\right)|10\rangle \\
\left.\cos \left(\frac{\pi}{4}-\theta\right)|10\rangle \pm \sin \left(\frac{\pi}{4}-\theta\right)|01\rangle\right) \\
\cos \left(\frac{\pi}{4}-\theta\right)|11\rangle+e^{-i \varphi} \sin \left(\frac{\pi}{4}-\theta\right)|00\rangle
\end{array}\right) .
$$

Hence with the concept of the Bloch vectors on the Bloch sphere [1, the variables $\theta$ and $\varphi$ realize their geometric meanings and the construction of the CNOT gate becomes clear.

To obtain other two-qubit quantum gates, for instance the CNOT gate, we have to apply single qubit unitary transformations $A, B, C, D$ which can be possibly found in the Bloch sphere [1] by $S O(3)$ rotations, namely,

$$
(A \otimes B) U_{ \pm}(\theta)(C \otimes D)=P_{\uparrow} \otimes \mathbb{1}+P_{\downarrow} \otimes \sigma_{x}=\mathrm{CNOT}
$$

which yields the CNOT gate and where the states $|\uparrow\rangle$ and $|\downarrow\rangle$ are the eigenvectors of $\sigma_{z}, \sigma_{z}|\uparrow\rangle=|\uparrow\rangle, \sigma_{z}|\downarrow\rangle=-|\downarrow\rangle$ and the projection operators $P_{\uparrow}$ and $P_{\downarrow}$ have the forms

$$
P_{\uparrow}=|\uparrow\rangle\left\langle\uparrow\left|, \quad P_{\downarrow}=\right| \downarrow\right\rangle\langle\downarrow| .
$$

Define the $S O(3)$ rotation around the $\vec{n}$-axis by

$$
D_{\vec{n}}(\theta)=e^{-\frac{i}{2}(\vec{\sigma} \cdot \vec{n}) \theta}
$$

where $\vec{\sigma}=\left(\sigma_{x}, \sigma_{y}, \sigma_{z}\right)$. For examples:

$$
D_{z}\left(-\frac{\varphi}{2}\right)=e^{i \frac{\varphi}{4} \sigma_{z}}, D_{x}\left(\frac{\pi}{2}\right)=e^{-i \frac{\pi}{4} \sigma_{x}}, D_{y}\left(\frac{\pi}{2}\right)=e^{-i \frac{\pi}{4} \sigma_{y}}
$$

satisfy

$$
D_{x}\left(\frac{\pi}{2}\right) D_{z}\left(-\frac{\varphi}{2}\right) \sigma_{n_{1}} D_{z}\left(\frac{\varphi}{2}\right) D_{x}\left(-\frac{\pi}{2}\right)=\sigma_{z}, \quad D_{z}\left(-\frac{\varphi}{2}\right) \sigma_{n_{2}} D_{z}\left(\frac{\varphi}{2}\right)=\sigma_{x} .
$$

Consider the time-evolution operator $U_{+}(\theta)$ (114). Choosing suitable single qubit transformations, we obtain

$$
\left(D_{x}\left(\frac{\pi}{2}\right) D_{z}\left(-\frac{\varphi}{2}\right) \otimes D_{z}\left(-\frac{\varphi}{2}\right)\right) U_{+}(\theta)\left(D_{z}\left(\frac{\varphi}{2}\right) D_{x}\left(-\frac{\pi}{2}\right) \otimes D_{z}\left(\frac{\varphi}{2}\right)\right)=e^{-\frac{i}{2}\left(\sigma_{z} \otimes \sigma_{x}\right) \theta}
$$


which has another form

$$
e^{-\frac{i}{2}\left(\sigma_{z} \otimes \sigma_{x}\right) \theta}=P_{\uparrow} \otimes e^{-\frac{i}{2} \sigma_{x} \theta}+P_{\downarrow} \otimes e^{\frac{i}{2} \sigma_{x} \theta} .
$$

Set $\theta=\frac{\pi}{2}$. To construct the CNOT gate, we need additional single qubit transformations

$$
\left(\delta \otimes e^{i \frac{\pi}{4} \sigma_{x}}\right) e^{-i \frac{\pi}{4}\left(\sigma_{z} \otimes \sigma_{x}\right)}=\mathrm{CNOT}
$$

in which the phase gate $\delta$ has the form $\delta=P_{\uparrow}-i P_{\downarrow}$, see (133).

Consider the time-evolution operator $U_{-}\left(\theta=-\frac{\pi}{2}, \varphi=0\right)$, namely the unitary $\check{R}$-matrix (132) given by $e^{i \frac{\pi}{4}\left(\sigma_{x} \otimes \sigma_{y}\right)}$ which is transformed into $e^{i \frac{\pi}{4}\left(\sigma_{z} \otimes \sigma_{x}\right)}$ by

$$
\left(D_{y}\left(-\frac{\pi}{2}\right) \otimes D_{z}\left(-\frac{\pi}{2}\right)\right) e^{i \frac{\pi}{4}\left(\sigma_{x} \otimes \sigma_{y}\right)}\left(D_{y}\left(\frac{\pi}{2}\right) \otimes D_{z}\left(\frac{\pi}{2}\right)\right)=e^{i \frac{\pi}{4}\left(\sigma_{z} \otimes \sigma_{x}\right)} .
$$

So we obtain another proof for Theorem 1 [10].

\section{Concluding remarks}

Motivated by the observation that there are certain natural similarities between quantum entanglements and topological entanglements, we derive the unitary solutions of the QYBE or the unitary $\check{R}(x)$-matrices via Yang-Baxterization and construct the related Hamiltonians for the standard and non-standard representations of the six-vertex model and the complete solutions of the non-vanishing eight-vertex model. With the Brylinksis' theorem [13], the unitary $\check{R}(x)$-matrix is also a universal quantum gate except very special cases.

The remark has to be made on the classification of the unitary $\check{R}(x)$-matrices we obtained. We classify them according to Yang-Baxterization. We don't try to classify them with other approaches since we can't obtain the complete solutions of the QYBE (8) only via Yang-Baxterization. But the unitary solutions of the Yang-Baxter equation (the braid relation) have been classified [12. For example, the first type of solution for the eight-vertex model in our case belongs the fourth family specified by [12]. But for the eight-vertex model, we have three types solutions of the BGR (7) but have four types of the unitary $\check{R}(x)$-matrices.

It is worthwhile arguing again that quantum entanglements for quantum information processing are related to not only topological entanglements but also geometric invariants. The previous one leads us to viewing the unitary braiding operator as a quantum entanglement operator and a universal quantum gate, while the latter one suggests us to regard unitary $\check{R}(x)$-matrices as universal quantum gates. In addition, it is important to mention another view of topological issues for quantum computing in terms of anyonic models, see [42, 43, 44, 45, 46, 47].

\section{Acknowledgements}

Y. Zhang is indebted to X.Y. Li for his constant encouragement, guidance and support during the research. He thanks the hospitality of Institut des Hautes 
Études Scientifiques, Bures-sur-Yvette during the stay and the hospitality of Institut für Theoretical Physik, Leipzig University during the stay. This work is in part supported by NSFC-10447134.

For L.H. Kauffman, most of this effort was sponsored by the Defense Advanced Research Projects Agency (DARPA) and Air Force Research Laboratory, Air Force Materiel Command, USAF, under agreement F30602-01-2-05022. The U.S. Government is authorized to reproduce and distribute reprints for Government purposes notwithstanding any copyright annotations thereon. The views and conclusions contained herein are those of the authors and should not be interpreted as necessarily representing the official policies or endorsements, either expressed or implied, of the Defense Advanced Research Projects Agency, the Air Force Research Laboratory, or the U.S. Government. (Copyright 2004.) It gives L.H. Kauffman great pleasure to acknowledge support from NSF Grant DMS-0245588.

\section{A A practical revisit to Yang-Baxterization}

Yang-Baxterization [24] is a prescription deriving solutions to the QYBE (8) from the BGR (7). We consider a BGR $b$-matrix with two distinct non-vanishing eigenvalues $\lambda_{1}$ and $\lambda_{2}$ taking the form

$$
b=\lambda_{1} P_{1}+\lambda_{2} P_{2}
$$

where $P_{1}$ and $P_{2}$ are the projection matrices satisfying

$$
P_{1}+P_{2}=\mathbb{1}, \quad P_{1}^{2}=P_{1}, P_{2}^{2}=P_{2}, P_{1} P_{2}=0 .
$$

With the help of Yang-Baxterization, the corresponding $\check{R}(x)$-matrix modulo an overall scalar factor [28, 29] has the form

$$
\begin{aligned}
\check{R}(x) & =\left(\lambda_{1}+\lambda_{2} x\right) P_{1}+\left(\lambda_{2}+\lambda_{1} x\right) P_{2} \\
& =b+\lambda_{1} \lambda_{2} x b^{-1}
\end{aligned}
$$

where the inverse matrix $b^{-1}$ is given by

$$
b^{-1}=\frac{1}{\lambda_{1}} P_{1}+\frac{1}{\lambda_{2}} P_{2}
$$

In the case that the BGR $b$-matrix has three distinct non-vanishing eigenvalues $\lambda_{1}, \lambda_{2}$ and $\lambda_{3}$, Yang-Baxterization [28, 29] leads to the following formula

$$
\check{R}(x)=\lambda_{1} \lambda_{3} x(x-1) b^{-1}+\left(\lambda_{1}+\lambda_{2}+\lambda_{3}+\lambda_{1} \lambda_{3} \lambda_{2}^{-1}\right) x \mathbb{1}-(x-1) b .
$$

Changing the ordering of three different eigenvalues in principle gives us different solutions of the QYBE (8). However, this formula is symmetric with respect to interchanging $\lambda_{1}$ and $\lambda_{3}$, so usually we obtain three types of the $\check{R}(x)$-matrices. 
With two formulas (150) and (152), the $\check{R}(x)$-matrix is proportional to the unit matrix $\mathbb{1}$ at $x=1$, namely,

$$
\check{R}(x=1) \propto \mathbb{1}
$$

except the case of $\lambda_{1}+\lambda_{2}+\lambda_{3}+\lambda_{1} \lambda_{3} \lambda_{2}^{-1}=0$. Hence for simplicity, in the following we will not pay special attention to the case of $x=1$.

One remark has to be made. The formula (150) has been proved to satisfy the QYBE (8), however the formula (152) does not have the general proof verifying that it is the solution of the QYBE (8). Once the $\check{R}(x)$-matrix is obtained via Yang-Baxterization, it would be safest to check whether it truly satisfies the QYBE (8).

\section{A.1 Yang-Baxterization of the six-vertex model}

Consider a non-standard BGR $b$-matrix suitable for constructing the Alexander polynomial [2]

$$
b=\left(\begin{array}{cccc}
q & 0 & 0 & 0 \\
0 & 0 & 1 & 0 \\
0 & 1 & q-q^{-1} & 0 \\
0 & 0 & 0 & -q^{-1}
\end{array}\right)
$$

where the deformation parameter $q$ has been assumed to be non-vanishing. It has two distinct eigenvalues: $q$ and $-q^{-1}$. In terms of the projection matrices $P_{1}(q)$ and $P_{2}(q)$ :

$$
P_{1}(q)=\left(\begin{array}{cccc}
1 & 0 & 0 & 0 \\
0 & \frac{1}{1+q^{2}} & \frac{q}{1+q^{2}} & 0 \\
0 & \frac{q}{1+q^{2}} & \frac{q^{2}}{1+q^{2}} & 0 \\
0 & 0 & 0 & 0
\end{array}\right), \quad P_{2}(q)=\left(\begin{array}{cccc}
0 & 0 & 0 & 0 \\
0 & \frac{q^{2}}{1+q^{2}} & \frac{-q}{1+q^{2}} & 0 \\
0 & \frac{-q}{1+q^{2}} & \frac{1}{1+q^{2}} & 0 \\
0 & 0 & 0 & 1
\end{array}\right)
$$

the BGR $b$-matrix is also given by

$$
b=q P_{1}(q)-q^{-1} P_{2}(q) .
$$

With the help of Yang-Baxterization, the BGR $b$-matrix corresponds to the following $\check{R}(x)$-matrix satisfying the QYBE (8),

$$
\begin{aligned}
\check{R}(x) & =\left(q-q^{-1} x\right) P_{1}(q)+\left(-q^{-1}+q x\right) P_{2}(q) \\
& =b-x b^{-1} .
\end{aligned}
$$

\section{A.2 Yang-Baxterization of the eight-vertex model}

The eight-vertex model assumes a general form

$$
b=\left(\begin{array}{cccc}
w_{1} & 0 & 0 & w_{7} \\
0 & w_{5} & w_{3} & 0 \\
0 & w_{4} & w_{6} & 0 \\
w_{8} & 0 & 0 & w_{2}
\end{array}\right)
$$


in terms of non-vanishing Boltzman weights $w_{i}, i=1, \cdots 8$. We will present the complete solutions of the BGR (7) for the non-vanishing eight-vertex model and construct the corresponding $\check{R}(x)$-matrices via Yang-Baxterization.

Introduce $f_{\alpha \beta \gamma}^{\kappa \lambda \omega}$ by

$$
f_{\alpha \beta \gamma}^{\kappa \lambda \omega}=\sum_{\mu \nu \rho}\left(b_{\alpha \beta}^{\mu \nu} b_{\nu \gamma}^{\rho \kappa} b_{\mu \rho}^{\lambda \omega}-b_{\beta \gamma}^{\mu \nu} b_{\alpha \mu}^{\lambda \rho} b_{\rho \nu}^{\omega \kappa}\right)
$$

where indices take spin up or spin down, namely \pm sign or spins $\pm \frac{1}{2}$. With the above ansatz, we have $b_{\alpha \beta}^{\kappa \omega}=0$ for $\alpha+\beta \neq \kappa+\omega \bmod 2$. In the case of $\alpha+\beta+\gamma \neq \lambda+\omega+\kappa \bmod 2$, the braid relation (7) is satisfied automatically, $f_{\alpha \beta \gamma}^{\lambda \omega \kappa}=0$. Hence we have to treat thirty-two equations of the Boltzman weights.

We solve all possible equations obtained by substituting the above ansatz of the eight-vertex model into the braid relation (7). We observe the equation $\left(w_{5}-w_{6}\right) w_{7} w_{8}=0$ showing $w_{5}=w_{6}$. With the equations $\left(w_{3}-w_{4}\right)\left(w_{1}-w_{5}\right) w_{8}=$ 0 and $\left(w_{3}-w_{4}\right)\left(w_{2}-w_{5}\right) w_{7}=0$, we have to choose either $w_{3}=w_{4}, w_{5}=w_{6}$ or $w_{3} \neq w_{4}, w_{5}=w_{1}=w_{2}=w_{6}$.

Setting $w_{5}=w_{1}=w_{2}=w_{6}$ gives us $w_{1}^{2}=w_{3}^{2}=w_{4}^{2}$ and $w_{3}^{2}+w_{7} w_{8}=0$. In the case of $w_{3} \neq w_{4}$, we have $w_{3}=-w_{4}$ and $w_{1}= \pm w_{3}$. The BGR $b_{ \pm}$-matrices have the forms

$$
b_{ \pm}=\left(\begin{array}{cccc}
w_{1} & 0 & 0 & w_{7} \\
0 & w_{1} & \pm w_{1} & 0 \\
0 & \mp w_{1} & w_{1} & 0 \\
-\frac{w_{1}^{2}}{w_{7}} & 0 & 0 & w_{1}
\end{array}\right) \Longleftrightarrow\left(\begin{array}{cccc}
1 & 0 & 0 & q \\
0 & 1 & \pm 1 & 0 \\
0 & \mp 1 & 1 & 0 \\
-q^{-1} & 0 & 0 & 1
\end{array}\right) .
$$

It has two eigenvalues $\lambda_{1}=1-i, \lambda_{2}=1+i$. The corresponding $\check{R}(x)$-matrices via Yang-Baxterization are obtained to be

$$
\check{R}_{ \pm}(x)=b_{ \pm}+2 x b_{ \pm}^{-1} .
$$

Imposing $w_{5}=w_{6}$ and $w_{3}=w_{4}$ in the given eight-vertex model, the solutions of the QYBE (8) have to satisfy the following three independent equations [29],

$$
\begin{aligned}
w_{5}^{2}-w_{7} w_{8} & =0 \\
w_{1}^{2}-w_{3}^{2}-w_{1} w_{5}+w_{2} w_{5} & =0 \\
w_{2}^{2}-w_{3}^{2}+w_{1} w_{5}-w_{2} w_{5} & =0 .
\end{aligned}
$$

Since the $\check{R}(x)$-matrix is modulo an overall scalar factor, we introduce

$$
q=\frac{w_{7}}{w_{5}}, \quad q^{-1}=\frac{w_{8}}{w_{5}}, \quad t=\frac{w_{2}}{w_{5}}, \quad z=\frac{w_{3}}{w_{5}}
$$

and rewrite the above equations in an explicit way as

$$
\begin{aligned}
t^{2}+\left(\frac{w_{1}}{w_{5}}\right)^{2} & =2 z^{2}, \\
\left(\frac{w_{1}}{w_{5}}-t\right)\left(\frac{w_{1}}{w_{5}}+t-2\right) & =0 .
\end{aligned}
$$


Therefore we obtain two types of the BGR (7). The first one is given by taking $\frac{w_{1}}{w_{5}}=2-t$ and the second one is specified by $\frac{w_{1}}{w_{5}}=t$.

In the first case of $\frac{w_{1}}{w_{5}}=2-t$, set $z=\left(t^{2}-2 t+2\right)^{\frac{1}{2}}$. The BGR $b_{ \pm}$-matrices take the form

$$
b_{ \pm}=\left(\begin{array}{cccc}
2-t & 0 & 0 & q \\
0 & 1 & \pm z & 0 \\
0 & \pm z & 1 & 0 \\
q^{-1} & 0 & 0 & t
\end{array}\right)
$$

It has two distinct eigenvalues $1 \pm z$ and so via Yang-Baxterization the $\check{R}(x)$ matrices are obtained by

$$
\check{R}_{ \pm}(x)=b_{ \pm}+x\left(1-z^{2}\right) b_{ \pm}^{-1}
$$

where the matrix entries satisfy $w_{1} w_{2}+w_{3} w_{4}=w_{5} w_{6}+w_{7} w_{8}$.

In the second case of $w_{3}=w_{4}$, namely take $\frac{w_{1}}{w_{5}}=t$ so that $z= \pm t$. The corresponding BGR $b_{ \pm}$-matrix has the form

$$
b_{ \pm}=\left(\begin{array}{cccc}
t & 0 & 0 & q \\
0 & 1 & \pm t & 0 \\
0 & \pm t & 1 & 0 \\
q^{-1} & 0 & 0 & t
\end{array}\right)
$$

It has three eigenvalues $1+t, 1-t$ and $-1+t$. To apply the formula (152) for the case of three distinct eigenvalues, we arrange three eigenvalues $\lambda_{1}, \lambda_{2}$ and $\lambda_{3}$ in three different orderings as follows

$$
\begin{array}{lccc} 
& \lambda_{1} & \lambda_{2} & \lambda_{3} \\
\text { the first ordering: } & 1+t & 1-t & t-1 \\
\text { the second ordering: } & 1+t & t-1 & 1-t \\
\text { the third ordering: } & 1-t & 1+t & t-1
\end{array} .
$$

For the first ordering and the second one, we have

$$
\lambda_{1}+\lambda_{2}+\lambda_{3}+\lambda_{1} \lambda_{3} \lambda_{2}^{-1}=0
$$

so that the situation goes back to the case of two distinct eigenvalues,

$$
\begin{aligned}
\check{R}_{ \pm}(x) & =-(x-1)\left(b_{ \pm} \pm x\left(1-t^{2}\right) b_{ \pm}^{-1}\right) \\
& \propto b_{ \pm} \pm x\left(1-t^{2}\right) b_{ \pm}^{-1}
\end{aligned}
$$

where the plus is for the first ordering and the minus is for the second ordering. We only need discuss the case of the first ordering.

For the BGR $b$-matrix (167) having three distinct eigenvalues, with the first ordering, we have

$$
\check{R}_{ \pm}(x)=b_{ \pm}+x\left(1-t^{2}\right) b_{ \pm}^{-1}
$$

with the third ordering (168), we have

$$
\check{R}_{ \pm}(x)=-(x-1) \lambda_{2}\left(b_{ \pm}-\lambda_{1} \lambda_{3} x b_{ \pm}^{-1}\right)+\left(\lambda_{2}^{2}+\lambda_{1} \lambda_{3}\right) x \mathbb{1} .
$$




\section{References}

[1] M. Nielsen and I. Chuang, Quantum Computation and Quantum Information (Cambridge University Press, 1999).

[2] L.H. Kauffman, Knots and Physics (World Scientific Publishers, 2002).

[3] P.K. Aravind, Borromean Entanglement of the GHZ state, in Potentiality, Entanglement and Passion-at-a-Distance, Cohen, Robert S., M. Horne, and J. Stachel (eds.), Kluwer Academic Publishers, Boston 1997.

[4] L.H. Kauffman, Quantum Computation and the Jones Polynomial, in Quantum Computation and Information, S. Lomonaco, Jr. (ed.), AMS CONM/305, 2002, pp. 101-137.

[5] L.H. Kauffman and S.J. Lomonaco Jr., New J. Phys. 4 (2002) 73.1-73.18.

[6] L.H. Kauffman and S.J. Lomonaco Jr., Entanglement Criteria-Quantum and Topological, in Quantum Information and Computation - Spie Proceedings, 21-22 April, 2003, Orlando, FL, ed. by E. Donkor, A.R. Pirich and H.E. Brandt, Volume 5105, pp. 51-58.

[7] L.H. Kauffman, Quantum Topology and Quantum Computing, in Quantum Computation, S. Lomonaco (ed.), AMS PSAPM/58, 2002, pp. 273-303.

[8] L. H. Kauffman and S. J. Lomonaco Jr., Quantum Knots, in Quantum Information and Computation II, Spie Proceedings, 12 -14 April 2004, Orlando, $F L$, ed. by E. Donkor, A.R. Pirich and H.E. Brandt, Volume 5436, pp. 268284.

[9] L.H. Kauffman, Opt. Spectrosc. 9 (2005) 227-232. Arxiv: quan-ph/0407224.

[10] L.H. Kauffman and S.J. Lomonaco Jr., New J. Phys. 6 (2004) 134. Arxiv: quant-ph/0401090.

[11] Y. Zhang, L.H. Kauffman and M.L. Ge, Universal Quantum Gate, YangBaxterization and Hamiltonian. To appear in Int. J. Quant. Inform. 4 (2005), Arxiv: quant-ph/0412095

[12] H. Dye, Quant. Inf. Proc. 2 (2003) 117-150.

[13] J.L. Brylinski and R. Brylinski, Universal quantum gates, in Mathematics of Quantum Computation, Chapman \& Hall/CRC Press, Boca Raton, Florida, 2002 (edited by R. Brylinski and G. Chen).

[14] C.N. Yang, Phys. Rev. Lett. 19 (1967) 1312-1314.

[15] R.J. Baxter, Annals Phys. 70 (1972) 193-228. 
[16] L.D. Faddeev, Integrable Models in (1+1)-Dimensional Quantum Field Theory, in Proc. of Summer School of Theoretical Physics, Les Houches, 1982 (Elsevier, Amsterdam, 1984).

[17] M. Jimbo, Adv. Ser. Math. Phys. 10 (1989) 1.

[18] V.G. Drinfeld, Sov. Math. Dokl. 32 (1985) 254.

[19] M. Jimbo, Int. J. Mod. Phys. A 4 (1989) 3759.

[20] A.A. Belavin and V.G. Drinfeld, Funcl. Anal. Appl. 16 (1982) 159.

[21] N.Yu. Reshetikhin, Quantized Universal Enveloping Algebras, the YangBaxter Equation and Invariants Of Links. 1, LOMI-E-4-87 (1988).

[22] N.Yu. Reshetikhin, Quantized Universal Enveloping Algebras, the YangBaxter Equation and Invariants Of Links. 2, LOMI-E-17-87 (1988).

[23] M. Jimbo, Commun. Math. Phys. 102 (1986) 537.

[24] V.F.R. Jones, Int. J. Mod. Phys. A 6 (1991) 2035-2043.

[25] Y. Akutsu and M. Wadati, J. Phys. Soc. Jap. 56 (1987) 3039-3051.

[26] Y. Akutsu, T. Deguchi and M. Wadati, J. Phys. Soc. Jap. 56 (1987) 34643479 .

[27] V.G. Turaev, Invent. Math. 92 (1988) 527.

[28] M.L. Ge, K. Xue and Y.S. Wu, Yang-Baxterization and Algebraic Structures, eds. C.N. Yang and M.L. Ge (World Scientific, Singpore, 1989).

[29] Y. Cheng, M.L. Ge and K. Xue, Commun. Math. Phys. 136 (1991) 195.

[30] M.L. Ge, Y.S. Wu and K. Xue, Int. J. Mod. Phys 6 A (1991) 3735.

[31] K. Sogo, M. Uchinami, Y. Akutsu and M. Wadati, Prog. Theor. Phys. 68 (1982).

[32] M.L. Ge, L. H. Gwa and H. K. Zhao, J. Phys. A: Math. Gen. 23 (1990) L 795-L 798.

[33] M. Couture, Y. Cheng, M.L. Ge and K. Xue, Int. J. Mod. Phys. 6 A (1991) 559.

[34] N.H. Jing, M.L. Ge and Y.S. Wu, Lett. Math. Phys. 21 (1991) 183.

[35] M.L. Ge and K. Xue, J. Math. Phys. 32 (1991) 1301-1309.

[36] H.C. Lee, Physics, Geometry, and Topology, Proceedings of the NATO Advanced Study Institute and Banff Summer School in Theoretical Physics (Plemum. N.Y. 1991). 
[37] H.C. Lee and M. Couture, A Method to Construct Closed Braids from Links and a New Polynomial for Connected Links, Chalk River Preprint, Canada (1988).

[38] C.L. Schultz, Phys. Rev. Lett. 46 (1981) 629.

[39] R.F. Werner, Phys. Rev. A 40 (1989) 4277.

[40] S. Hill and W.K. Wootters, Phys. Rev. Lett. 78 (1997) 5022-5025.

[41] W.K. Wootters, Phys. Rev. Lett. 80 (1998) 2245-2248.

[42] M. Freedman, Comm. Math. Phys. 234 (2003) 129-183.

[43] M. Freedman, Topological Views on Computational Complexity, Documenta Mathematica-Extra Volume ICM (1998) 453-464.

[44] M. Freedman, M. Larsen, and Z. Wang, Comm. Math. Phys. 227 (2002) 605-622.

[45] M. H. Freedman, A. Kitaev, Z. Wang, Commun. Math. Phys. 227 (2002) 587-603.

[46] M. Freedman, Found. Comput. Math. 1 (2001) 183-204.

[47] M. Bordewich, M. Freedman, L. Lovasz and D. Welsh, Approximate Counting and Quantum Computation (Combinatorics, Probability and Computing, Cambridge University Press). 